Homology, Homotopy and Applications, vol.17(1), 2015, pp.377-400

\title{
MOTIVIC LANDWEBER EXACT THEORIES AND THEIR EFFECTIVE COVERS
}

\author{
MARC LEVINE \\ (communicated by Charles A. Weibel)
}

\begin{abstract}
Let $k$ be a field of characteristic 0 , and let $(F, R)$ be a Landweber exact formal group law. We consider a Landweber exact $T$-spectrum $\mathcal{E}:=R \otimes_{\mathbb{L}}$ MGL and its effective cover $f_{0} \mathcal{E} \rightarrow$ $\mathcal{E}$ with respect to Voevodsky's slice tower. The coefficient ring $R_{0}$ of $f_{0} \mathcal{E}$ is the subring of $R$ consisting of elements of $R$ of nonpositive degree; the power series $F \in R[[u, v]]$ has coefficients in $R_{0}$, although $\left(F, R_{0}\right)$ is not necessarily Landweber exact. We show that the geometric part $X \mapsto f_{0} \mathcal{E}^{*}(X):=\left(f_{0} \mathcal{E}\right)^{2 *, *}(X)$ of $f_{0} \mathcal{E}$ is canonically isomorphic to the oriented cohomology theory $X \mapsto R_{0} \otimes_{\mathbb{L}} \Omega^{*}(X)$, where $\Omega^{*}$ is the theory of algebraic cobordism as defined in [12]. This recovers results of Dai-Levine [2] as the special case of algebraic $K$-theory and its effective cover, connective algebraic $K$-theory.
\end{abstract}

\section{Introduction}

Let $S$ be a fixed base-scheme, $\mathbf{S m} / S$ the category of smooth quasi-projective $S$ schemes, and $\mathcal{S H}(S)$ the motivic stable homotopy category of $T$-spectra. In this paper we consider two types of cohomology theories, which carry the designation "oriented." The first type are those bi-graded theories on $\mathrm{Sm} / S, X \mapsto \mathcal{E}^{*, *}(X)$ represented by a (weak) commutative ring $T$-spectrum $\mathcal{E} \in \mathcal{S H}(S)$ with an orientation $c$ in the reduced cohomology $\tilde{\mathcal{E}}^{2,1}\left(\mathbb{P}^{\infty}\right)$. The second are the oriented theories in the sense of [12, Definition 1.1.1], that is, contravariant functors $X \mapsto A^{*}(X)$ from $\mathbf{S m} / S$ to commutative graded rings, together with push-forward maps $f_{*}$ for projective morphisms $f: Y \rightarrow X$, satisfying a number of functorialities and compatibilities. We will refer to the first type as "motivic" theories, the second as "geometric." Assigning to a motivic theory $\mathcal{E}^{*, *}$ its geometric part $X \mapsto \mathcal{E}^{2 *, *}(X)$ gives the link between these two notions.

Among the oriented motivic theories, the theory represented by Voevodsky's algebraic cobordism spectrum MGL is the universal one (see [28] for the construction of MGL, [21] for its universality). For $S=\operatorname{Spec} k, k$ a field of characteristic 0, algebraic cobordism $\Omega^{*}$ as defined in $[\mathbf{1 2}]$ is the universal geometric theory.

The author thanks the Humboldt Foundation for support through the Humboldt Professorship. Received July 16, 2014, revised December 30, 2014; published on May 18, 2015.

2010 Mathematics Subject Classification: Primary 14C25, 19E15; Secondary 19E08, 14F42, 55P42.

Key words and phrases: Algebraic cobordism, oriented theory, slice tower.

Article available at http://dx.doi.org/10.4310/HHA.2015.v17.n1.a18

Copyright (C) 2015, International Press. Permission to copy for private use granted. 
For $S=\operatorname{Spec} k$ as above, the universal property of $\Omega^{*}$ gives a canonical natural transformation $\Omega^{*} \rightarrow \mathrm{MGL}^{2 * * *}$ of geometric theories. Relying on results of Hopkins, Morel, and Hoyois $[\mathbf{5}, \mathbf{6}]$, this was shown to be an isomorphism in $[\mathbf{1 1}]$. The main purpose of this paper is to extend this isomorphism property to other motivic theories and their geometric parts.

For a formal group law $(F, R), F(u, v) \in R[[u, v]]$, one can form the oriented geometric theory $X \mapsto R \otimes_{\mathbb{L}} \Omega^{*}(X)$, where $\mathbb{L}$ is the Lazard ring and $\mathbb{L} \rightarrow R$ the classifying homomorphism for $F$. In the motivic setting, the situation is more delicate, however, just as in the classical case, if $(F, R)$ is a Landweber exact formal group law, there is a corresponding oriented weak commutative ring spectrum $\mathrm{MGL}(R)$ with $\operatorname{MGL}(R)^{*, *}(X) \cong R \otimes_{\mathbb{L}} \operatorname{MGL}^{*, *}(X)$ (see [19]).

One can go a bit farther by considering the effective cover $f_{0} \mathcal{E} \rightarrow \mathcal{E}$ of a $T$-spectrum $\mathcal{E} \in \mathcal{S H}(S)$. Here, $f_{0}$ is the truncation functor with respect to Voevodsky's slice tower. $f_{0} \mathcal{E}$ is an analog of the classical -1 connective cover of a spectrum, and inherits many properties from $\mathcal{E}$. In particular, for $\mathcal{E}$ an oriented weak commutative ring $T$ spectrum, the effective cover $f_{0} \mathcal{E}$ inherits from $\mathcal{E}$ a canonical structure of an oriented weak commutative ring $T$-spectrum; the coefficient ring $R_{0}:=\left(f_{0} \mathcal{E}\right)^{2 *, *}(S)$ is just the degree $\leqslant 0$ part of the coefficient $\operatorname{ring} R:=\mathcal{E}^{*, *}(S)$ of $\mathcal{E}$ (at least for $S$ the spectrum of a characteristic 0 field; see Theorem 3.9). The coefficients of the group law $F_{\mathcal{E}}$ associated to $\mathcal{E}$ actually lie in $R_{0}$, but even if $\left(F_{\mathcal{E}}, R\right)$ is Landweber exact, it is usually the case that $\left(F_{\mathcal{E}}, R_{0}\right)$ is not.

For $\mathcal{E}$ the oriented spectrum $\operatorname{MGL}(R)$ associated to a Landweber exact formal group law, with effective cover $\mathcal{E}_{0}$, our main result is the following: For $k$ a characteristic 0 field, $S=$ Spec $k$, the canonical natural transformations

$$
\mathcal{E}^{2 *, *}(k) \otimes_{\mathbb{L}^{*}} \Omega^{*} \rightarrow \mathcal{E}^{2 *, *}, \mathcal{E}_{0}^{2 *, *}(k) \otimes_{\mathbb{L}^{*}} \Omega^{*} \rightarrow\left(f_{0} \mathcal{E}\right)^{2 *, *}
$$

are isomorphisms of geometric oriented cohomology theories on $\mathbf{S m} / k$. We actually prove a stronger result (see Corollary 6.3) concerning the oriented Borel-Moore homology theories on $\mathbf{S c h} / k$ defined by $\mathcal{E}$ and $f_{0} \mathcal{E}$.

The case of the Landweber exact theory $\mathcal{E}^{*, *}$ follows immediately from the case $\mathcal{E}=$ MGL, proved in $[\mathbf{1 1}]$, so our efforts are directed at the effective cover $\mathcal{E}_{0}$. The main idea for these results already appears in our treatment (with S. Dai) of the case of algebraic $K$-theory and its effective cover [2]. We axiomatize the situation via the notion of a geometrically Landweber exact motivic oriented theory (see Definition 3.7) and show that for such a theory $\mathcal{E}$, the canonical map

$$
\mathcal{E}^{2 *, *}(k) \otimes_{\mathbb{L}^{*}} \Omega^{*}(X) \rightarrow \mathcal{E}^{2 *, *}(X)
$$

is an isomorphism for all $X \in \mathbf{S m} / k$ (Theorem 6.2). Our result on a Landweber exact theory and its effective cover then follow once we show that these are both geometrically Landweber exact (Theorem 3.9).

The paper is organized as follows: We begin by recalling some of the basic notions concerning oriented (weak) commutative ring spectra in the motivic stable homotopy category in $\S 1$, where we also recall the main results on the universality of MGL. In $\S 2$ we recall basic facts about the slice tower in the motivic stable homotopy category; we discuss as well some issues of convergence of the slice spectral sequence. In $\S 3$ we introduce the effective cover of an oriented weak ring $T$-spectrum and show that it too defines an oriented weak commutative ring spectrum. We discuss Landweber 
exact theories and introduce the notion of a geometrically Landweber exact theory. We discuss oriented duality theories on the category of smooth pairs over $k$ in $\S 4$. This theory provides the link between geometric theories and motivic theories via the operation of taking the geometric part of a motivic oriented theory. We describe the relation of oriented duality theories with algebraic cobordism in $\S 5$ and put everything together and prove our main results in the final section, $\S 6$.

I would like to thank the referee for a number of helpful comments and suggestions.

Notation and conventions. We let $\mathbf{S p c}$ and $\mathbf{S p c} \bullet$ denote the categories of simplicial sets and pointed simplicial sets, respectively, with homotopy categories $\mathcal{H}$ and $\mathcal{H}_{\bullet}$. Spt is the category of spectra (for the usual suspension operator $\Sigma:=(-) \wedge S^{1}$ ) and $\mathcal{S H}$ is the stable homotopy category.

We denote by $\mathbb{G}_{m}$ the pointed $S$-scheme $\left(\mathbb{A}_{S}^{1}-0_{S}, 1_{S}\right) . \mathbb{P}_{*}^{n}$ will denote the pointed $S$-scheme $\mathbb{P}_{S}^{n}$, with base-point $[1,0, \ldots, 0]$; we similarly give the limit $\mathbb{P}^{\infty}$ the basepoint $[1,0, \ldots]$.

We use a base-scheme $S$ that is separated, regular, noetherian and of finite Krull dimension; we will at a certain point specialize to the case $S=\operatorname{Spec} k, k$ a field of characteristic $0 . \mathbf{S c h} / S$ will denote the category of quasi-projective schemes over $S$ and $\mathbf{S m} / S$ the full subcategory of smooth, quasi-projective schemes over $S . \operatorname{Spc}(S)$ and Spc. $(S)$ are, respectively, the categories of presheaves on $\mathbf{S m} / S$ with values in Spc and $\mathbf{S p c} . \mathbf{S p t}_{S^{1}}(S)$ will denote the category of $S^{1}$-spectra over $S$, this being the category of presheaves of spectra on $\mathbf{S m} / S$. We let $\mathbf{S p t}_{T}(S)$ denote the category of $T$-spectra in Spc. $(S)$, with $T:=\mathbb{A}^{1} / \mathbb{A}^{1} \backslash\{0\}$.

The categories $\mathbf{S p c}(S), \mathbf{S p c}(S), \mathbf{S p t}_{S^{1}}(S)$ and $\mathbf{S p t}_{T}(S)$ all have motivic model structures (the original source for the unstable theory is $[\mathbf{1 5}]$; see also $[\mathbf{3}, \mathbf{4}]$ for a compact description. For the stable theory, we refer the reader to $[\mathbf{7}])$, with homotopy categories denoted $\mathcal{H}(S), \mathcal{H}_{\bullet}(S), \mathcal{S H}_{S^{1}}(S)$ and $\mathcal{S H}(S)$, respectively. The categories $\mathcal{S H}_{S^{1}}(S)$ and $\mathcal{S H}(S)$ are triangulated tensor categories with translation given by $S^{1}$ suspension; the tensor structure is constructed using symmetric spectrum versions of $\mathbf{S p t}_{S^{1}}(S)$ and $\mathbf{S} \mathbf{S t}_{T}(S)$, with appropriate model structures, also discussed in [7]. The unit for the tensor structure in $\mathcal{S H}(S)$ is the motivic sphere spectrum $\mathbb{S}_{S}:=\Sigma_{T}^{\infty} S_{+}$.

We have suspension functors $\Sigma^{a, b}: \mathcal{S H}(S) \rightarrow \mathcal{S H}(S)$ defined for $a \geqslant b \geqslant 0$ by $\Sigma^{a, b}(\mathcal{E})=\mathcal{E} \wedge S^{a-b} \wedge \mathbb{G}_{m}^{\wedge b}$, and extending to $a, b \in \mathbb{Z}$ using the invertibility of $\Sigma^{1,0}$ and $\Sigma^{1,1}$. We have as well the $T$-suspension $\Sigma_{T}(\mathcal{E}):=\mathcal{E} \wedge T$, canonically isomorphic to $\Sigma^{2,1}$. In addition, we have infinite suspension functors $\Sigma_{T}^{\infty}: \mathcal{H} \bullet(S) \rightarrow \mathcal{S H}(S)$, $\Sigma_{s}^{\infty}: \mathcal{H}_{\bullet}(S) \rightarrow \mathcal{S H}_{S^{1}}(S)$ and $\Sigma_{\mathbb{G}_{m}}^{\infty}: \mathcal{S H}_{S^{1}}(S) \rightarrow \mathcal{S H}(S)$ with respective right adjoints $\Omega_{T}^{\infty}: \mathcal{S H}(S) \rightarrow \mathcal{H}_{\bullet}(S), \Omega_{S^{1}}^{\infty}: \mathcal{S H}_{S^{1}}(S) \rightarrow \mathcal{H}_{\bullet}(S)$ and $\Omega_{\mathbb{G}_{m}}^{\infty}: \mathcal{S H}(S) \rightarrow \mathcal{S H}_{S^{1}}(S)$.

We let $\mathbb{L}$ denote the Lazard ring, that is, the coefficient ring of the universal rank 1 commutative formal group law $F_{\mathbb{L}} \in \mathbb{L}[[u, v]]$. We let $\mathbb{L}^{*}$ denote $\mathbb{L}$ with the grading determined by $\operatorname{deg} a_{i j}=1-i-j$ if $F_{\mathbb{L}}(u, v)=u+v+\sum_{i, j \geqslant 1} a_{i j} u^{i} v^{j}$ and let $\mathbb{L}_{*}$ denote $\mathbb{L}$ with the opposite grading $\mathbb{L}_{n}:=\mathbb{L}^{-n}$.

\section{1. $\quad$ Oriented ring $T$-spectra}

We recall that a morphism $f: \mathcal{E} \rightarrow \mathcal{F}$ in a compactly generated triangulated category $\mathcal{T}$ is a phantom map if for each compact object $A$ in $\mathcal{T}$, the induced map 
$f_{*}: \operatorname{Hom}_{\mathcal{T}}(A, \mathcal{E}) \rightarrow \operatorname{Hom}_{\mathcal{T}}(A, \mathcal{F})$ is zero; it is enough to check on compact objects of the form $\mathcal{X}[n]$, where $\mathcal{X}$ is an element in a given set of compact generators and $n \in \mathbb{Z}$. The subset of phantom maps $\operatorname{Hom}_{\mathcal{T}}(\mathcal{E}, \mathcal{F})_{\mathrm{ph}} \subset \operatorname{Hom}_{\mathcal{T}}(\mathcal{E}, \mathcal{F})$ is clearly a twosided ideal, so we may form the category $\mathcal{T} / \mathrm{ph}$ with the same objects as $\mathcal{T}$ and morphisms $\operatorname{Hom}_{\mathcal{T} / \mathrm{ph}}(\mathcal{E}, \mathcal{F}):=\operatorname{Hom}_{\mathcal{T}}(\mathcal{E}, \mathcal{F}) / \operatorname{Hom}_{\mathcal{T}}(\mathcal{E}, \mathcal{F})_{\mathrm{ph}}$.

We will be mainly interested in the case $\mathcal{T}=\mathcal{S H}(S)$, in which the shifted suspension spectra $\Sigma_{T}^{n} \Sigma_{T}^{\infty} X_{+}, X \in \mathbf{S m} / S, n \in \mathbb{Z}$, form a set of compact generators. Thus, two maps $f, g: \mathcal{E} \rightarrow \mathcal{F}$ are equal modulo phantom maps if and only if $f_{*}=g_{*}$ as maps of the associated bi-graded cohomology theories $f_{*}, g_{*}: \mathcal{E}^{*, *} \rightarrow \mathcal{F}^{*, *}$ on $\mathbf{S m} / S$.

Definition 1.1. A commutative ring $T$-spectrum is a $T$-spectrum $\mathcal{E} \in \mathbf{S p t}_{T}(S)$ together with maps $\mu: \mathcal{E} \wedge \mathcal{E} \rightarrow \mathcal{E}, 1: \mathbb{S}_{S} \rightarrow \mathcal{E}$ in $\mathcal{S H}(S)$ such that $(\mathcal{E}, \mu, 1)$ is a commutative monoid in $\mathcal{S H}(S)$. A weak commutative ring $T$-spectrum is a $T$-spectrum $\mathcal{E} \in$ $\operatorname{Spt}_{T}(S)$ together with maps $\mu: \mathcal{E} \wedge \mathcal{E} \rightarrow \mathcal{E}, 1: \mathbb{S}_{S} \rightarrow \mathcal{E}$ in $\mathcal{S H}(S)$ such that $(\mathcal{E}, \mu, 1)$ is a commutative monoid in $\mathcal{S H}(S) / \mathrm{ph}$.

For $\mathcal{E}, \mathcal{F}$ (weak) commutative ring $T$-spectra, a morphism $f: \mathcal{E} \rightarrow \mathcal{F}$ in $\mathcal{S H}(S)$ is a monoid map (resp. weak monoid map) if $f$ is a map of monoid objects in $\mathcal{S H}(S)$ (resp. in $\mathcal{S H}(S) / \mathrm{ph})$.

Definition 1.2. An orientation on a weak commutative ring $T$-spectrum $(\mathcal{E}, \mu, 1)$ is an element $c \in \mathcal{E}^{2,1}\left(\mathbb{P}_{*}^{\infty}\right)$ such that the restriction $c_{\mid \mathbb{P}^{1}} \in \mathcal{E}^{2,1}\left(\mathbb{P}_{*}^{1}\right)$ corresponds to $1 \in \mathcal{E}^{0,0}(S)$ under the suspension isomorphism $\mathcal{E}^{0,0}(S) \cong \mathcal{E}^{2,1}(T) \cong \mathcal{E}^{2,1}\left(\mathbb{P}_{*}^{1}\right)$.

A pair $(\mathcal{E}, c)$ consisting of a weak commutative ring $T$-spectrum $(\mathcal{E}, \mu, 1)$ and an orientation $c$ is an oriented weak commutative ring $T$-spectrum. We say that a weak commutative ring $T$-spectrum $(\mathcal{E}, \mu, 1)$ is orientable if there is an orientation $c$ on $\mathcal{E}$. We sometimes omit the explicit mention of the orientation $c$.

A morphism $f:(\mathcal{E}, c) \rightarrow\left(\mathcal{E}^{\prime}, c^{\prime}\right)$ of oriented weak commutative ring $T$-spectra is a morphism $f: \mathcal{E} \rightarrow \mathcal{E}^{\prime}$ in $\mathcal{S H}(S)$ such that $f$ is a weak monoid map and $f_{*}(c)=c^{\prime}$.

Example 1.3. The algebraic cobordism spectrum MGL has been studied in [21]. MGL is the $T$-spectrum $\left(\mathrm{MGL}_{0}, \mathrm{MGL}_{1}, \ldots\right)$ with $\mathrm{MGL}_{n}$ the Thom space $T h\left(E_{n}\right)$, with $E_{n} \rightarrow B \mathrm{GL}_{n}$ the universal $n$-plane bundle. $\mathrm{MGL}_{S}$ is a commutative ring $T$-spectrum in $\mathcal{S H}(S)$ with an orientation $c_{\mathrm{MGL}} \in \mathrm{MGL}^{2,1}\left(\mathbb{P}_{*}^{\infty}\right)$ given by noting that the diagram

$$
\mathbb{P}^{\infty} \longleftarrow E_{1} \longrightarrow T h\left(E_{1}\right)=\mathrm{MGL}_{1}
$$

induces an isomorphism $\mathbb{P}_{*}^{\infty} \cong \mathrm{MGL}_{1}$ in $\mathcal{H}_{\bullet}(S)$ and thereby a morphism $c_{\mathrm{MGL}}$ : $\Sigma_{T}^{\infty} \mathbb{P}_{*}^{\infty} \rightarrow \Sigma^{2,1} \mathrm{MGL}$ in $\mathcal{S H}(S)$.

We recall the following result of Panin, Pimenov, and Röndigs.

Theorem 1.4 ([21, Theorem 1.1]). For $\mathcal{E}$ a commutative ring $T$-spectrum in $\mathcal{S H}(S)$, sending a monoid morphism $\varphi: \mathrm{MGL} \rightarrow \mathcal{E}$ to $\varphi\left(c_{\mathrm{MGL}}\right)$ gives a bijection of the set of monoid maps $\varphi$ with the set of orientations $c_{\mathcal{E}} \in \mathcal{E}^{2,1}\left(\mathbb{P}_{*}^{\infty}\right)$.

Given an oriented commutative ring $T$-spectrum $(\mathcal{E}, c)$ in $\mathcal{S H}(S)$, we let

$$
\varphi_{\mathcal{E}, c}: \mathrm{MGL} \rightarrow \mathcal{E}
$$

denote the corresponding morphism of commutative $\operatorname{ring} T$-spectra. 
In fact, this result extends directly to the setting of oriented weak commutative ring spectra, replacing "monoid map" with "weak monoid map." Indeed, the proof in $[\mathbf{2 1}]$ reduces to proving certain identities in $\mathcal{E}^{*, *}(\operatorname{MGL}(n))$ or $\mathcal{E}^{*, *}(\operatorname{MGL}(n) \wedge \operatorname{MGL}(n))$. It is shown in $[\mathbf{2 1}]$ that the canonical map

$$
\mathcal{E}^{*, *}(\operatorname{MGL}(n)) \rightarrow \lim _{\leftarrow} \mathcal{E}^{*, *}(\operatorname{Th}(\mathcal{T}(n, m)))
$$

is an isomorphism, where $\mathcal{T}(n, m) \rightarrow \operatorname{Grass}(n, n+m)$ is the universal bundle and $\operatorname{Th}(-)$ is the Thom space. Similarly,

$$
\mathcal{E}^{*, *}(\operatorname{MGL}(n) \wedge \operatorname{MGL}(n)) \cong \lim _{\leftarrow} \mathcal{E}^{*, *}(\operatorname{Th}(\mathcal{T}(n, m)) \wedge \operatorname{Th}(\mathcal{T}(n, m))) .
$$

Their proof relies on showing that the maps on $\mathcal{E}$-cohomology in the inverse system are surjective, hence the proofs only require the knowledge of $\mathcal{E}$-cohomology on objects in $\mathbf{S m} / S$, and therefore the proofs work for oriented weak commutative ring spectra without change.

In case $S=\operatorname{Spec} k, k$ a field, Vezzosi's proof of the universality of MGL [25, Theorem 4.3] is also based on obtaining identities in $\mathcal{E}^{* *}(\operatorname{MGL}(n))$ or $\mathcal{E}^{* *}(\operatorname{MGL}(n) \wedge$ $\operatorname{MGL}(n))$ and thus can also be adapted to the setting of oriented weak commutative $\operatorname{ring} T$-spectra.

Remark 1.5. Let $\mathcal{E} \in \mathcal{S H}(S)$ be a weak commutative ring $T$-spectrum. Let $t_{\mathcal{E}} \in$ $\mathcal{E}^{1,1}\left(\mathbb{G}_{m}\right)$ be the element corresponding to the unit $1 \in \mathcal{E}^{0,0}(S)$ under the suspension isomorphism. By functoriality, $t_{\mathcal{E}}$ gives a map of pointed sets

$$
t_{\mathcal{E}}(X): \mathcal{O}_{X}^{\times}(X) \rightarrow \mathcal{E}^{1,1}(X)
$$

If $\mathcal{E}$ admits an orientation $c_{\mathcal{E}} \in \mathcal{E}^{2,1}\left(\mathbb{P}_{*}^{\infty}\right)$, then $t_{\mathcal{E}}(X)$ is a group homomorphism. ${ }^{1}$ Using the $\mathcal{E}^{*, *}(S)$-module structure on $\mathcal{E}^{*, *}(X), t_{\mathcal{E}}(X)$ extends to a map of $\mathcal{E}^{*, *}(S)$ modules

$$
t_{\mathcal{E}}(X): \mathcal{E}^{2 *, *}(S) \otimes_{\mathbb{Z}} \mathcal{O}_{X}^{\times}(X) \rightarrow \mathcal{E}^{2 *+1, *+1}(X) .
$$

\section{The slice spectral sequence}

Voevodsky introduced in [26] his slice tower as a motivic analog to the classical Postnikov tower for spectra. In addition to $[\mathbf{2 6}]$, we refer the reader to $[\mathbf{2 7}]$ and $[\mathbf{8}, \mathbf{9}]$ for the basic facts concerning the slice tower, some of which we briefly recall here for the reader's convenience.

We consider the localizing subcategory $\Sigma_{T}^{p} \mathcal{S} \mathcal{H}^{\text {eff }}(S)$ of $\mathcal{S H}(S)$ generated by objects $\Sigma_{T}^{n} X_{+}$, with $X \in \mathrm{Sm} / S$ and $n \geqslant p$, with inclusion $i_{p}: \Sigma_{T}^{p} \mathcal{S} \mathcal{H}^{\text {eff }}(S) \rightarrow \mathcal{S H}(S)$. The objects $\Sigma_{T}^{n} X_{+}$are compact, hence each of the categories $\Sigma_{T}^{p} \mathcal{S} \mathcal{H}^{\text {eff }}(S)$ is compactly generated; the set of all $\Sigma_{T}^{n} X_{+}, n \in \mathbb{Z}, X \in \mathbf{S m} / S$ similarly forms a set of compact generators for $\mathcal{S H}(S)$.

Thanks to results of Neeman [17] on compactly generated triangulated categories, the functor $i_{p}$ admits a right adjoint $r_{p}: \mathcal{S H}(S) \rightarrow \Sigma_{T}^{p} \mathcal{S H} \mathcal{H}^{\text {eff }}(S)$. Defining $f_{p}:=i_{p} \circ$

\footnotetext{
${ }^{1}$ Letting $\mathbb{S}$ denote the sphere spectrum and writing $[a]:=t_{\mathbb{S}}(a)$, this follows from the identity $[a b]=[a]+[b]+\eta[a][b]\left(\eta: \mathbb{S} \wedge \mathbb{G}_{m} \rightarrow \mathbb{S}\right.$ the stable Hopf map) and the fact that $\eta$ goes to zero in any oriented theory $\mathcal{E}$. Both these facts are proven by Morel in $[\mathbf{1 4}, \S 6]$.
} 
$r_{p}: \mathcal{S H}(S) \rightarrow \mathcal{S H}(S)$, one has canonical natural transformations $f_{p} \rightarrow \mathrm{id}, f_{p+1} \rightarrow f_{p}$. Applying these to a $T$-spectrum $\mathcal{E} \in \mathcal{S H}(S)$ yields the the slice tower

$$
\ldots \rightarrow f_{p+1} \mathcal{E} \rightarrow f_{p} \mathcal{E} \rightarrow \ldots \rightarrow \mathcal{E} .
$$

The slice functor $s_{p}: \mathcal{S H}(S) \rightarrow \mathcal{S H}(S)$ is characterized up to unique isomorphism by natural transformations $f_{p} \rightarrow s_{p} \rightarrow f_{p+1}[1]$ so that for each $\mathcal{E} \in \mathcal{S H}(S)$, the triangle

$$
f_{p+1} \mathcal{E} \rightarrow f_{p} \mathcal{E} \rightarrow s_{p} \mathcal{E} \rightarrow f_{p+1} \mathcal{E}[1]
$$

is distinguished.

There are canonical isomorphisms for all $a, b, p$,

$$
\Sigma^{a, b} \circ f_{p} \cong f_{p+b} \circ \Sigma^{a, b} ; \quad \Sigma^{a, b} \circ s_{p} \cong s_{p+b} \circ \Sigma^{a, b},
$$

compatible with the defining distinguished triangles for the $s_{p}$. The canonical map $f_{n} \mathcal{E} \rightarrow \mathcal{E}$ induces an isomorphism $\Omega_{\mathbb{G}_{m}}^{\infty} f_{n} \mathcal{E} \cong \Omega_{\mathbb{G}_{m}}^{\infty} \mathcal{E}$ in $\mathcal{S H}_{S^{1}}(S)$ for all $n \leqslant 0$.

For $Y \in \mathbf{S m} / S$, we have the associated slice spectral sequence

$$
E_{2}^{p, q}(Y ; n)=\left(s_{-q} \mathcal{E}\right)^{p+q, n}(Y) \Longrightarrow \mathcal{E}^{p+q, n}(Y) .
$$
(2.1).

We conclude this section with a convergence criterion for the spectral sequence

Lemma 2.1. Suppose that $S=\operatorname{Spec} k, k$ a perfect field. Take $\mathcal{E} \in \mathcal{S H}(S)$. Suppose that there is a non-decreasing function $f: \mathbb{Z} \rightarrow \mathbb{Z}$ with $\lim _{n \rightarrow \infty} f(n)=\infty$, such that $\pi_{a+b, b} \mathcal{E}=0$ for $a \leqslant f(b)$. Then the for all $Y$, the spectral sequence (2.1) is strongly convergent. ${ }^{2}$

Proof. We may rewrite the $E_{2}$-term in (2.1) as

$$
E_{2}^{p, q}(Y ; n)=\operatorname{Hom}_{\mathcal{S H}(S)}\left(\Sigma_{T}^{\infty} Y_{+}, \Sigma^{p+q, n} s_{-q} \mathcal{E}\right) .
$$

The spectral sequence will be strongly convergent (for fixed $n$ ) if for each $m_{0} \in \mathbb{Z}$, there is an integer $q\left(m_{0}\right)$ such that

$$
\operatorname{Hom}_{\mathcal{S H}(S)}\left(\Sigma_{T}^{\infty} Y_{+}, \Sigma^{m, n} f_{q} \mathcal{E}\right)=0
$$

for all $q \geqslant q\left(m_{0}\right)$ and all $m \geqslant-m_{0}$. Indeed, we have

$$
\begin{aligned}
\operatorname{Hom}_{\mathcal{S H}(S)}\left(\Sigma_{T}^{\infty} Y_{+}, \Sigma^{m, n} f_{q} \mathcal{E}\right) & =\operatorname{Hom}_{\mathcal{S} \mathcal{H}_{S^{1}}(S)}\left(\Sigma_{S^{1}}^{-m} \Sigma_{S^{1}}^{\infty} Y_{+}, \Omega_{\mathbb{G}_{m}}^{\infty} \Sigma^{0, n} f_{q} \mathcal{E}\right) \\
& =\pi_{-m}\left(\Omega_{\mathbb{G}_{m}}^{\infty} \Sigma^{0, n} f_{q} \mathcal{E}(Y)\right) .
\end{aligned}
$$

Thus, the above condition is just saying that, given an integer $m_{0}$, the spectrum $\Omega_{\mathbb{G}_{m}}^{\infty} \Sigma^{0, n} f_{q} \mathcal{E}(Y)$ is $m_{0}$-connected for all $q \geqslant q\left(m_{0}\right)$, this being a standard criterion for the strong convergence of the spectral sequence associated to the tower of spectra

$$
\ldots \rightarrow \Omega_{\mathbb{G}_{m}}^{\infty} \Sigma^{0, n} f_{q} \mathcal{E}(Y) \rightarrow \ldots \rightarrow \Omega_{\mathbb{G}_{m}}^{\infty} \Sigma^{0, n} f_{-n} \mathcal{E}(Y) \cong \Omega_{\mathbb{G}_{m}}^{\infty} \Sigma^{0, n} \mathcal{E}(Y)
$$

We proceed to exhibit the existence of such an integer $q\left(m_{0}\right)$.

\footnotetext{
${ }^{2}$ As spectral sequence $\left\{E_{r}^{p q}\right\} \Rightarrow G^{p+q}$ converges strongly to $G^{*}$ if for each $n$, the spectral sequence filtration $F^{*} G^{n}$ on $G^{n}$ is finite and exhaustive, there is an $r(n)$ such that for all $p$ and all $r \geqslant r(n)$, all differentials entering and leaving $E_{r}^{p, n-p}$ are zero and the resulting maps $E_{r}^{p, n-p} \rightarrow E_{\infty}^{p, n-p}=$ $\mathbf{G r}_{F}^{p} G^{n}$ are all isomorphisms.
} 
We recall the 2-variable Postnikov tower $f_{a, b}$ defined in $[\mathbf{8}, \S 3]$, with $f_{b}=f_{-\infty, b}$. We also recall that

i) $\pi_{m+r, r} f_{a, b} \mathcal{F}=0$ for $m<a, b, r \in \mathbb{Z}[\mathbf{8}$, Lemma 4.4],

ii) the canonical map $\rho_{a, b}: f_{a, b} \mathcal{F} \rightarrow f_{b} \mathcal{F}$ induces an isomorphism on $\pi_{m+r, r}$ for all $m \geqslant a, r \geqslant b$, and

iii) $\rho_{a, b}: f_{a, b} \mathcal{F} \rightarrow f_{b} \mathcal{F}$ is an isomorphism if $\rho$ induces an isomorphism on $\pi_{m+r, r}$ for all $m \in \mathbb{Z}$ and $r \geqslant b$ [8, Lemma 4.6].

The universal property of $f_{q+n}$ gives the isomorphism for all $r \geqslant q+n$

$$
\pi_{m+r, r} f_{q+n} \Sigma^{0, n} \mathcal{E} \cong \pi_{m+r, r} \Sigma^{0, n} \mathcal{E} \cong \pi_{m+r, r-n} \mathcal{E} .
$$

By assumption on $\mathcal{E}, \pi_{m+n+r-n, r-n} \mathcal{E}=0$ for $m+n \leqslant f(r-n)$, and thus

$$
\pi_{m+r, r} f_{q+n}\left(\Sigma^{0, n} \mathcal{E}\right)=0
$$

for $m \leqslant f(r-n)-n$ as well (assuming $r \geqslant q+n$ ).

Now take $q\left(m_{0}\right)$ to be an integer such that $f\left(q\left(m_{0}\right)\right) \geqslant m_{0}+\operatorname{dim} Y+n$, and take $q \geqslant q\left(m_{0}\right)$. As $f$ is non-decreasing, we have $f(r-n)-n \geqslant f\left(q\left(m_{0}\right)\right)-n \geqslant m_{0}+$ $\operatorname{dim} Y$ for all $r \geqslant q+n$, and hence

$$
\pi_{m+r, r} f_{q+n}\left(\Sigma^{0, n} \mathcal{E}\right)=0
$$

for $m \leqslant m_{0}+\operatorname{dim} Y, r \geqslant q+n$. But by (i)-(iii), this implies that the map

$$
f_{m_{0}+\operatorname{dim} Y+1, q+n}\left(\Sigma^{0, n} \mathcal{E}\right) \rightarrow f_{q+n}\left(\Sigma^{0, n} \mathcal{E}\right)
$$

is an isomorphism, and thus by (i), $\pi_{a+b, b} f_{q+n}\left(\Sigma^{0, n} \mathcal{E}\right)=0$ for all $a \leqslant m_{0}+\operatorname{dim} Y$, $b \in \mathbb{Z}$. Translating back to $\Omega_{\mathbb{G}_{m}}^{\infty} \Sigma^{0, n} f_{q} \mathcal{E}$ via the isomorphisms

$$
\pi_{a+b, b} f_{q+n}\left(\Sigma^{0, n} \mathcal{E}\right) \cong \pi_{a+b, b} \Sigma^{0, n} f_{q} \mathcal{E} \cong \pi_{a+b, b} \Omega_{\mathbb{G}_{m}}^{\infty} \Sigma^{0, n} f_{q} \mathcal{E}
$$

for $b \geqslant 0, a \in \mathbb{Z}$, we have

$$
\pi_{a+b, b} \Omega_{\mathbb{G}_{m}}^{\infty} \Sigma^{0, n} f_{q} \mathcal{E}=0
$$

for all $a \leqslant m_{0}+\operatorname{dim} Y, b \geqslant 0, q \geqslant q\left(m_{0}\right)$. In particular, taking $b=0$,

$$
\pi_{a} \Omega_{\mathbb{G}_{m}}^{\infty} \Sigma^{0, n} f_{q} \mathcal{E}=0
$$

for all $a \leqslant m_{0}+\operatorname{dim} Y, q \geqslant q\left(m_{0}\right)$.

We now apply the local-global spectral sequence (with $q \geqslant q\left(m_{0}\right)$ )

$$
E_{2}^{a, b}=H^{a}\left(Y_{\mathrm{Nis}}, \pi_{-b} \Omega_{\mathbb{G}_{m}}^{\infty} \Sigma^{0, n} f_{q} \mathcal{E}\right) \Longrightarrow \pi_{-a-b}\left(\Omega_{\mathbb{G}_{m}}^{\infty} \Sigma^{0, n} f_{q} \mathcal{E}(Y)\right) .
$$

This sequence is convergent by [1, Theorem 8] since $Y$ has finite Nisnevich cohomological dimension $\leqslant \operatorname{dim} Y$ and is strongly convergent since $\pi_{-b} \Omega_{\mathbb{G}_{m}}^{\infty} \Sigma^{0, n} f_{q} \mathcal{E}=0$ for $-b \leqslant$ $m_{0}+\operatorname{dim} Y$. In particular, $E_{2}^{a, b}=0$ for $a+b \geqslant-m_{0}$, and hence $\Omega_{\mathbb{G}_{m}}^{\infty} \Sigma^{0, n} f_{q} \mathcal{E}(Y)$ is $m_{0}$-connected for $q \geqslant q\left(m_{0}\right)$.

\section{Oriented theories and their $T$-effective covers}

3.1. The $T$-effective cover

Proposition 3.1. 1. Let $(\mathcal{E}, \mu, 1)$ be a weak commutative ring $T$-spectrum. Then $f_{0} \mathcal{E}$ has a unique structure of a weak commutative ring $T$-spectrum such that the canonical 
map $\rho: f_{0} \mathcal{E} \rightarrow \mathcal{E}$ is a weak monoid morphism.

2. If $(\mathcal{E}, c)$ is an oriented weak commutative ring $T$-spectrum, then there is a unique element $c_{0} \in\left(f_{0} \mathcal{E}\right)^{2,1}\left(\mathbb{P}_{*}^{\infty}\right)$ with $\rho_{*}\left(c_{0}\right)=c$, and $c_{0}$ defines an orientation on the weak commutative ring $T$-spectrum $f_{0} \mathcal{E}$.

3. We have $t_{\mathcal{E}}=\rho_{*}\left(t_{f_{0} \mathcal{E}}\right)$ in $\mathcal{E}^{1,1}\left(\mathbb{G}_{m}\right)$.

Proof. Write $\mathcal{E}_{0}$ for $f_{0} \mathcal{E}$. We first lift the multiplication. We note that $\mathcal{S} \mathcal{H}^{\mathrm{eff}}(S)$ is closed under smash product, as the generators are, so $\mathcal{E}_{0} \wedge \mathcal{E}_{0}$ is in $\mathcal{S} \mathcal{H}^{\text {eff }}(S)$. Thus, the composition

$$
\mathcal{E}_{0} \wedge \mathcal{E}_{0} \stackrel{\rho \wedge \rho}{\longrightarrow} \mathcal{E} \wedge \mathcal{E} \stackrel{\mu}{\rightarrow} \mathcal{E}
$$

admits a unique lifting $\mu_{0}: \mathcal{E}_{0} \wedge \mathcal{E}_{0} \rightarrow \mathcal{E}_{0}$ making

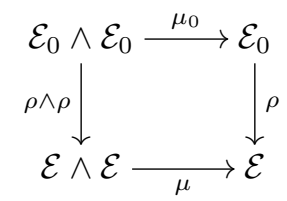

commute.

We claim that $\mu_{0}$ is associative modulo phantom maps. For this, let

$$
\varphi: \mathcal{E}_{0} \wedge \mathcal{E}_{0} \wedge \mathcal{E}_{0} \rightarrow \mathcal{E}_{0}
$$

denote the difference $\mu_{0} \circ\left(\mu_{0} \wedge\right.$ id $)-\mu_{0} \circ\left(\right.$ id $\left.\wedge \mu_{0}\right)$. Let $A$ be a compact object of $\mathcal{S H}(S)$ and let $h: A \rightarrow \mathcal{E}_{0} \wedge \mathcal{E}_{0} \wedge \mathcal{E}_{0}$ be a morphism in $\mathcal{S H}(S)$. We apply $[\mathbf{1 7}$, theorem 4.3.3] for the subcategory $\mathcal{S} \mathcal{H}^{\mathrm{eff}}(S)$ of $\mathcal{S H}(S)$, generated as a localizing subcategory of $\mathcal{S H}(S)$ by the set of compact objects $\mathcal{S}:=\left\{\Sigma_{T}^{\infty} X_{+}, X \in \mathbf{S m} / S\right\}$, with cardinal $\beta=$ $\aleph_{0}$. This tells us that there is a compact object $B$ in $\mathcal{S} \mathcal{H}^{\text {eff }}(S)$, and morphisms $f: A \rightarrow$ $B, g: B \rightarrow \mathcal{E}_{0} \wedge \mathcal{E}_{0} \wedge \mathcal{E}_{0}$, such that $h=g \circ f$. But as $B$ is compact, the composition $\rho \circ \varphi \circ g$ in $\mathcal{S H}(S)$ is zero, and since $B$ is in $\mathcal{S H}^{\text {eff }}(S)$, the universal property of $\rho: \mathcal{E}_{0} \rightarrow \mathcal{E}$ implies that $\varphi \circ g=0$ in $\mathcal{S H}^{\text {eff }}(S)$. Thus, $\varphi \circ h=\varphi \circ g \circ f=0$, and hence $\mu_{0}$ is associative modulo phantom maps.

The unit map 1: $\mathbb{S}_{S} \rightarrow \mathcal{E}$ factors uniquely through $\rho$, since $\mathbb{S}_{S}$ is in $\mathcal{S} \mathcal{H}^{\text {eff }}(S)$, giving the unit map $1_{0}: \mathbb{S}_{S} \rightarrow \mathcal{E}_{0}$; the identities

$$
\mu_{0} \circ\left(\operatorname{id}_{\mathcal{E}_{0}} \wedge 1_{0}\right)=\operatorname{id}_{\mathcal{E}_{0}}=\mu_{0} \circ\left(1_{0} \wedge \operatorname{id}_{\mathcal{E}_{0}}\right)
$$

in $\mathcal{S H}(S) /$ ph and the commutativity of $\mu_{0}$ modulo phantom maps follow as for the proof of associativity.

For $(2)$, recall that $*=[1,0, \ldots, 0]$ in $\mathbb{P}^{n}$. Let $\mathbb{A}^{n}(*) \subset \mathbb{P}^{n}$ be the open subscheme $X_{0} \neq 0$. Then the quotient map $\mathbb{P}_{*}^{n} \rightarrow \mathbb{P}^{n} / \mathbb{A}^{n}(*)$ is an isomorphism in $\mathcal{H}_{\bullet}(S)$, giving the isomorphism $\mathbb{P}_{*}^{\infty} \cong \lim _{n} \mathbb{P}^{n} / \mathbb{A}^{n}(*)$. In particular, both $\mathbb{P}_{*}^{n}$ and $\mathbb{P}_{*}^{\infty}$ are in $\Sigma_{T}^{1} \mathcal{S H}^{\mathrm{eff}}(S)$.

We have the isomorphism $\Sigma_{T} f_{0} \mathcal{E} \cong f_{1} \Sigma_{T} \mathcal{E}$, and under this isomorphism $\Sigma_{T} \rho: \Sigma_{T} f_{0} \mathcal{E} \rightarrow \Sigma_{T} \mathcal{E}$ goes over to the universal map $\rho_{1}: f_{1} \Sigma_{T} \mathcal{E} \rightarrow \Sigma_{T} \mathcal{E}$. Thus, $c: \mathbb{P}_{*}^{\infty} \rightarrow \Sigma_{T} \mathcal{E}$ factors uniquely through $\Sigma_{T} \rho$ via a map $c_{0}: \mathbb{P}_{*}^{\infty} \rightarrow \Sigma_{T} f_{0} \mathcal{E}$. The restriction of $c_{0}$ to $\mathbb{P}_{*}^{1}$ is similarly the unique lifting of $c_{\mid \mathbb{P}_{*}^{1}}$, which shows that $c_{0 \mid \mathbb{P}_{*}^{1}} \in$ $\left(f_{0} \mathcal{E}\right)^{2,1}\left(\mathbb{P}_{*}^{1}\right)$ corresponds to $1_{0} \in\left(f_{0} \mathcal{E}\right)^{0,0}(S)$ under the suspension isomorphism. Thus, $c_{0} \in\left(f_{0} \mathcal{E}\right)^{2,1}\left(\mathbb{P}_{*}^{\infty}\right)$ is an orientation for $f_{0} \mathcal{E}$. 
For (3), this follows directly from (1) and the naturality of the map $t_{\mathcal{E}}$ with respect to weak monoid morphisms.

\subsection{Landweber exact theories}

We recall the Landweber exactness conditions for a formal group law $(F, R)$ : Let $\varphi: \mathbb{L}^{*} \rightarrow R$ be the classifying map for $(F, R)$. Choose homogeneous polynomial generators for $\mathbb{L}^{*}$ over $\mathbb{Z}, \mathbb{L}^{*}=\mathbb{Z}\left[x_{1}, x_{2}, \ldots\right]$, with $\operatorname{deg} x_{i}=-i$. Then for each prime $p$, the sequence $p, x_{p-1}, \ldots, x_{p^{i}-1}, \ldots$ is a regular sequence on $R$.

For an oriented theory $(\mathcal{E}, c), \mathcal{E} \in \mathcal{S H}(S)$, let $R_{\mathcal{E}}^{*}$ be the coefficient ring, that is, $R_{\mathcal{E}}^{*}:=\mathcal{E}^{2 *, *}(S)$. Let $F_{\mathcal{E}, c}(u, v) \in R_{\mathcal{E}}^{*}[[u, v]]$ be the formal group law of the oriented theory $(\mathcal{E}, c)$.

We let $\mathcal{S H}(S)_{\mathcal{T}} \subset \mathcal{S H}(S)$ be the full subcategory of Tate spectra, that is, the localizing subcategory generated by the spheres $\Sigma^{a, b} \mathbb{S}_{S}, a, b \in \mathbb{Z}$. We recall from $[\mathbf{1 9}$, $\S 4]$ some basic facts concerning Tate spectra: There is an exact projection functor $p_{\mathcal{T}}: \mathcal{S H}(S) \rightarrow \mathcal{S H}(S)_{\mathcal{T}}$ right adjoint to the inclusion. For a morphism $f: S \rightarrow S^{\prime}$, the functor $L f^{*}: \mathcal{S H}\left(S^{\prime}\right) \rightarrow \mathcal{S H}(S)$ maps $\mathcal{S H}\left(S^{\prime}\right)_{\mathcal{T}}$ to $\mathcal{S H}(S)_{\mathcal{T}}$ and the restriction $L f_{\mathcal{T}}^{*}: \mathcal{S H}\left(S^{\prime}\right)_{\mathcal{T}} \rightarrow \mathcal{S H}(S)$ has right adjoint $p_{\mathcal{T}} \circ R f_{*}$. Finally, $p_{\mathcal{T}}$ is a $\mathcal{S H}(S)_{\mathcal{T}}$-module map, that is, for $\mathcal{E} \in \mathcal{S H}(S)_{\mathcal{T}}$ and $\mathcal{F} \in \mathcal{S H}(S)$, there is a natural isomorphism

$$
p_{\mathcal{T}}(\mathcal{E} \wedge \mathcal{F}) \cong \mathcal{E} \wedge p_{\mathcal{T}}(\mathcal{F}) .
$$

We let $\mathcal{S H}(S)_{\text {fin }} \subset \mathcal{S H}(S)$ denote the thick subcategory of finite spectra, namely, the thick subcategory generated by the spectra $\Sigma_{T}^{n} \Sigma_{T}^{\infty} X_{+}, X \in \mathbf{S m} / S, n \in \mathbb{Z}$. Similarly, we let $\mathcal{S H}(S)_{\mathcal{T} \text {,fin }} \subset \mathcal{S H}(S)_{\mathcal{T}}$ be the thick subcategory generated by spheres $\Sigma^{a, b} \mathbb{S}_{S}$, $a, b \in \mathbb{Z}$.

Definition 3.2. An oriented weak commutative ring $T$-spectrum $(\mathcal{E}, c)$ with classifying map $\varphi_{\mathcal{E}, c}:$ MGL $\rightarrow \mathcal{E}$ is said to be Landweber exact if

1 . The classifying map $\mathbb{L}^{*} \rightarrow R_{\mathcal{E}}^{*}$ for the formal group law $F_{\mathcal{E}, c}$ satisfies the Landweber exactness conditions.

2. For all finite spectra $\mathcal{F} \in \mathcal{S H}(S)_{\text {fin }}$, the map $R_{\mathcal{E}}^{*} \otimes_{R_{\mathrm{MGL}}^{*}} \operatorname{MGL}^{*, *}(\mathcal{F}) \rightarrow \mathcal{E}^{*, *}(\mathcal{F})$ induced by $\varphi_{\mathcal{E}, c}$ and the product map $\mathcal{E}^{2 n, n}(S) \otimes \mathcal{E}^{a, b}(\mathcal{F}) \rightarrow \mathcal{E}^{a+2 n, b+n}(\mathcal{F})$ is an isomorphism.

3. $\mathcal{E}$ is in the Tate subcategory $\mathcal{S H}(S)_{\mathcal{T}}$ of $\mathcal{S H}(S)$.

Remark 3.3. Let $k$ be a field of characteristic 0. Naumann, Spitzweck, and Østvær [19, theorem 8.7] show that, for each Landweber exact $\mathbb{L}^{*}$-algebra $R^{*}$, the bi-graded functor from finite spectra to bi-graded algebras, $\mathcal{F} \mapsto R^{*} \otimes_{\mathbb{L}^{*}} \mathrm{MGL}^{*, *}(\mathcal{F})$, is represented by an object $\operatorname{MGL}\left(R^{*}\right)$ in $\mathcal{S H}(k)_{\mathcal{T}}$ with morphisms $\mu: \operatorname{MGL}\left(R^{*}\right) \wedge \operatorname{MGL}\left(R^{*}\right) \rightarrow$ $\operatorname{MGL}\left(R^{*}\right), 1: \mathbb{S}_{k} \rightarrow \operatorname{MGL}\left(R^{*}\right)$ defining a (oriented) weak commutative ring $T$-spectrum. It does not seem to be known if one can give $\operatorname{MGL}\left(R^{*}\right)$ the structure of an oriented commutative ring $T$-spectrum.

In fact, Naumann, Spitzweck, and Østvær work in the setting of homology theories on $\mathcal{S H}(S)$ rather than cohomology theories on finite spectra, and they prove their results for $S$ a regular, noetherian separated scheme of finite Krull dimension. In case the base-scheme is Spec $k, k$ a field of characteristic 0, all finite spectra in $\mathcal{S H}(k)$ are strongly dualizable [23, Theorem 1.4], so one may easily pass from homology theories on $\mathcal{S H}(k)$ to cohomology theories on finite spectra in $\mathcal{S H}(k)$. 
Naumann, Spitzweck, and Østvær simply define a Landweber exact spectrum to be one of the form $\operatorname{MGL}\left(R^{*}\right)$, rather than giving an intrinsic characterization. In case $S=$ Spec $k, k$ a field of characteristic 0 , the construction of Naumann, Spitzweck, and Østvær in fact gives us all Landweber exact oriented weak commutative ring spectra, as the next two lemmas show.

Lemma 3.4. Suppose that $S=\operatorname{Spec} k, k$ a field of characteristic 0. Suppose that $(\mathcal{E}, c)$ is Landweber exact (in the sense of definition 3.2). Then the classifying map $\varphi_{\mathcal{E}, c}: \mathrm{MGL} \rightarrow \mathcal{E}$ and the product maps for $\mathcal{F} \in \mathcal{S H}(k), R_{*}^{\mathcal{E}} \otimes_{\mathbb{Z}} \mathcal{E}_{*, *}(\mathcal{F}) \rightarrow \mathcal{E}_{*, *}(\mathcal{F})$, induce an isomorphism of homology theories on $\mathcal{S H}(k)$

$$
R_{*}^{\mathcal{E}} \otimes_{R_{*}^{\mathrm{MGL}}} \mathrm{MGL}_{*, *}(-) \rightarrow \mathcal{E}_{*, *}(-) .
$$

Proof. Both functors $R_{*}^{\mathcal{E}} \otimes_{R_{*}^{\mathrm{MGL}}} \mathrm{MGL}_{*, *}(-)$ and $\mathcal{E}_{*, *}(-)$ are homological functors on $\mathcal{S H}(k)$, compatible with coproducts, hence the full subcategory $\mathcal{C}$ of objects $\mathcal{F}$ for which the lemma holds is a localizing subcategory of $\mathcal{S H}(k)$. As $\mathcal{S H}(k)$ is generated as a localizing category by $\mathcal{S H}(k)_{\text {fin }}$, this reduces us to the case $\mathcal{F} \in \mathcal{S H}(k)_{\text {fin }}$.

Take $\mathcal{F}$ in $\mathcal{S H}(k)_{\text {fin }}$. Then $\mathcal{F}$ is strongly dualizable with $\mathcal{F}^{D} \in \mathcal{S H}(k)_{\text {fin }}[\mathbf{2 3}$, Theorem 1.4], and we have $\mathcal{G}_{*, *}(\mathcal{F}) \cong \mathcal{G}^{-*,-*}\left(\mathcal{F}^{D}\right)$ for all $\mathcal{G} \in \mathcal{S H}(k)$. The fact that $\varphi_{\mathcal{E}, c}$ induces an isomorphism

$$
R_{\mathcal{E}}^{*} \otimes_{R_{\mathrm{MGL}}^{*}} \operatorname{MGL}^{*, *}\left(\mathcal{F}^{D}\right) \cong \mathcal{E}^{*, *}\left(\mathcal{F}^{D}\right) .
$$

shows that $\mathcal{S H}(k)_{\text {fin }} \subset \mathcal{C}$.

Lemma 3.5. Suppose $S=\operatorname{Spec} k, k$ a field of characteristic 0 . Let $(\mathcal{E}, c)$ be a Landweber exact oriented weak commutative ring $T$-spectrum (in the sense of definition 3.2). Then there is an isomorphism of oriented weak commutative ring T-spectra $\psi: \operatorname{MGL}\left(R_{\mathcal{E}}^{*}\right) \rightarrow \mathcal{E}$.

Proof. Since $k$ has characteristic 0 , the results of Hopkins, Morel, and Hoyois $[\mathbf{5 , 6}]$ show that the classifying map $\mathbb{L}_{*} \rightarrow R_{*}^{\mathrm{MGL}}$ is an isomorphism. We may therefore replace $R_{*}^{\mathrm{MGL}}$ with $\mathbb{L}_{*}$ in our definition of Landweber exactness and in Lemma 3.4.

Let $f: \operatorname{Spec} k \rightarrow \operatorname{Spec} \mathbb{Q}$ be the canonical morphism of schemes, giving the adjoint functors $L f^{*}: \mathcal{S H}(\mathbb{Q}) \rightleftarrows \mathcal{S H}(k): R f_{*}$. Consider the object $R f_{*} \mathcal{E} \in \mathcal{S H}(\mathbb{Z})$ and take $\mathcal{F} \in \mathcal{S H}(\mathbb{Z})_{\mathcal{T}}$. We have a canonical isomorphism $R f_{*}\left(\mathcal{E} \wedge L f^{*} \mathcal{F}\right) \cong R f_{*} \mathcal{E} \wedge \mathcal{F}$ (see, e.g., $[\mathbf{1 9}$, p. 578, eq. (26)]) and therefore by Lemma 3.4 we have

$$
\left(R f_{*} \mathcal{E}\right)_{*, *}(\mathcal{F})=\mathcal{E}_{*, *}\left(L f^{*} \mathcal{F}\right) \cong R_{*}^{\mathcal{E}} \otimes_{\mathbb{L}_{*}} \operatorname{MGL}_{k *, *}\left(L f^{*} \mathcal{F}\right)
$$

for all $\mathcal{F}$ in $\mathcal{S H}(\mathbb{Q})_{\mathcal{T}}$. That is, $R f_{*} \mathcal{E}$ represents the homology theory $\mathcal{F} \mapsto R_{*}^{\mathcal{E}} \otimes_{\mathbb{L} *}$ $\mathrm{MGL}_{k * * *}\left(L f^{*} \mathcal{F}\right)$ on $\mathcal{S H}(\mathbb{Q})_{\mathcal{T}}$; using the isomorphism (3.1), the same holds for the Tate projection $p_{\mathcal{T}} R f_{*} \mathcal{E}$. The spectrum $\operatorname{MGL}_{\mathbb{Q}}\left(R_{\mathcal{E}}^{*}\right) \in \mathcal{S H}(\mathbb{Q})$ similarly represents the homology theory $\mathcal{F} \mapsto R_{*}^{\mathcal{E}} \otimes_{\mathbb{L}_{*}} \operatorname{MGL}_{\mathbb{Q} *, *}(\mathcal{F})$.

We have a canonical isomorphism $\mathrm{MGL}_{k} \cong L f^{*} \mathrm{MGL}_{\mathbb{Q}}$; we consider this as an identity. The spectrum $\operatorname{MGL}_{k}\left(R_{\mathcal{E}}^{*}\right)$ is similary by construction canonically isomorphic to the pull-back $L f^{*} \mathrm{MGL}_{\mathbb{Q}}\left(R_{\mathcal{E}}^{*}\right)$. Applying $R f_{*}$ and the Tate projection to pair of canonical maps $\varphi_{\mathrm{MGL}\left(R_{\mathcal{E}}^{*}\right)}: \mathrm{MGL}_{k} \rightarrow \operatorname{MGL}_{k}\left(R_{\mathcal{E}}^{*}\right), \varphi_{\mathcal{E}}: \mathrm{MGL}_{k} \rightarrow \mathcal{E}$ and using the 
appropriate adjoint properties gives us the commutative diagram in $\mathcal{S H}(\mathbb{Q})$

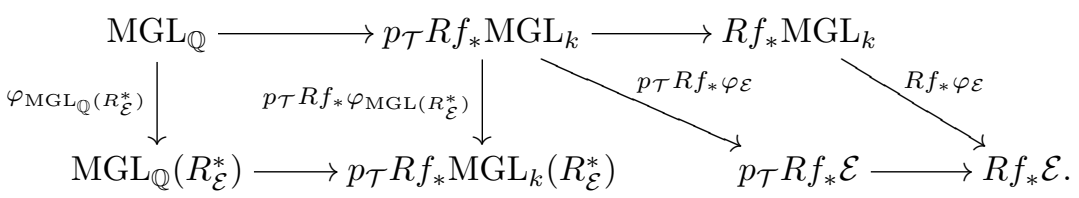

This induces the commutative diagram of homology theories on $\mathcal{S H}(\mathbb{Q})_{\mathcal{T}}$

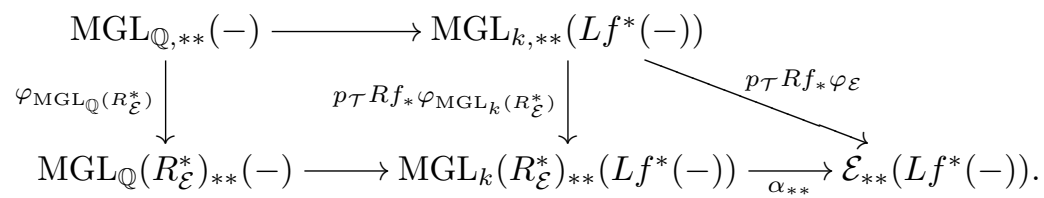

Here, $\alpha_{* *}$ is the isomorphism given by Lemma 3.4, and we use the identities

$$
\pi_{* *}\left(p_{\mathcal{T}}\left(R f_{*} \mathcal{F}\right) \wedge \mathcal{G}\right)(\mathbb{Q})=\pi_{* *}\left(R f_{*} \mathcal{F} \wedge \mathcal{G}\right)(\mathbb{Q})=\pi_{* *}\left(\mathcal{F} \wedge L f^{*} \mathcal{G}\right)(k)
$$

for $\mathcal{F} \in \mathcal{S H}(k), \mathcal{G} \in \mathcal{S H}(\mathbb{Q})_{\mathcal{T}}$.

As $\mathcal{S H}(\mathbb{Q})_{\mathcal{T}}$ satisfies Brown representability, in the sense of [16, Definition 3.1] (see [18, Theorem 1] and [16, Proposition 4.11 and Theorem 5.1]), the isomorphism $\alpha_{* *}$ arises from an isomorphism $\alpha: p_{\mathcal{T}} R f_{*} \operatorname{MGL}_{k}\left(R_{\mathcal{E}}^{*}\right) \rightarrow p_{\mathcal{T}} R f_{*} \mathcal{E}$ that fills in the diagram (3.2) to a commutative (up to phantoms) diagram. This gives us the diagram in $\mathcal{S H}(\mathbb{Q})$

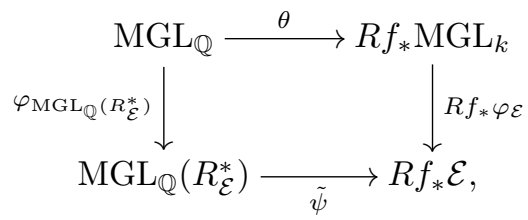

commutative after composition with a map $\mathcal{F} \rightarrow \mathrm{MGL}_{\mathbb{Q}}, \mathcal{F} \in \mathcal{S H}(\mathbb{Q})_{\mathcal{T} \text {, fin }}$, where $\theta$ is the unit of the adjunction and $\tilde{\psi}$ is the evident composition. Applying adjunction gives the diagram in $\mathcal{S H}(k)$

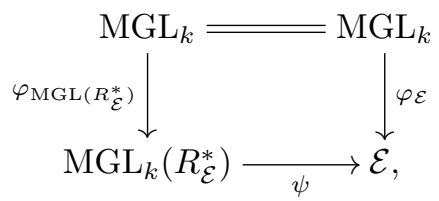

which we claim commutes up to a phantom map. Indeed, $\mathrm{MGL}_{\mathbb{Q}}$ is a filtered colimit of finite Tate spectra $F_{0} \mathrm{MGL}_{\mathbb{Q}} \rightarrow F_{1} \mathrm{MGL}_{\mathbb{Q}} \rightarrow \ldots \rightarrow \mathrm{MGL}_{\mathbb{Q}}$, with each of the maps $F_{n} \mathrm{MGL}_{\mathbb{Q}} \rightarrow F_{n+1} \mathrm{MGL}_{\mathbb{Q}}$ a cofibration of cofibrant objects in $\mathbf{S p t}_{T}(\mathbb{Q})_{\mathcal{T} \text {,fin }}$. This writes $\mathrm{MGL}_{k}=L f^{*} \mathrm{MGL}_{\mathbb{Q}}$ as the filtered colimit of finite Tate spectra $F_{0} \mathrm{MGL}_{k} \rightarrow$ $F_{1} \mathrm{MGL}_{k} \rightarrow \ldots \rightarrow \mathrm{MGL}_{k}$, by taking $F_{n} \mathrm{MGL}_{k} \cong L f^{*} F_{n} \mathrm{MGL}_{\mathbb{Q}}$. This gives us an isomorphism in $\mathcal{S H}(k)$

$$
\mathrm{MGL}_{k} \cong \underset{n}{\operatorname{hocolim}} F_{n} \mathrm{MGL}_{k}
$$

Let $i_{n}^{\mathbb{Q}}: F_{n} \mathrm{MGL}_{\mathbb{Q}} \rightarrow \mathrm{MGL}_{\mathbb{Q}}$ be the canonical map and let $i_{n}: F_{n} \mathrm{MGL}_{k} \rightarrow \mathrm{MGL}_{k}$ be the induced map. As $F_{n} \mathrm{MGL}_{\mathbb{Q}}$ is in $\mathcal{S H}(\mathbb{Q})_{\mathcal{T} \text {,fin }}$, we have $\tilde{\psi} \circ \varphi_{\mathrm{MGL}_{\mathbb{Q}}\left(R_{\mathcal{\varepsilon}}^{*}\right)} \circ i_{n}^{\mathbb{Q}}=$ 
$R f_{*} \varphi_{\mathcal{E}} \circ i_{n}^{\mathbb{Q}}$. Using adjointness again, we see that $\psi \circ \varphi_{\mathrm{MGL}_{k}\left(R_{\mathcal{E}}^{*}\right)} \circ i_{n}=\varphi_{\mathcal{E}} \circ i_{n}$ for all $n$. For $\mathcal{F} \in \mathcal{S H}(k)$ a compact object and $g: \mathcal{F} \rightarrow \mathrm{MGL}_{k}$ a morphism, $g$ factors through some $i_{n}$ and hence $\psi \circ \varphi_{\mathrm{MGL}_{k}\left(R_{\mathcal{E}}^{*}\right)} \circ g=\varphi_{\mathcal{E}} \circ g$, which verifes our claim.

As the maps

$$
\begin{gathered}
\cup \circ\left(i d \otimes \varphi_{\mathrm{MGL}_{k}\left(R_{\mathcal{E}}^{*}\right)}\right): R_{\mathcal{E}}^{*} \otimes_{\mathbb{L}^{*}} \operatorname{MGL}_{k}^{* *}(X) \rightarrow \operatorname{MGL}_{k}^{* *}\left(R_{\mathcal{E}}^{*}\right)(X) \\
\cup \circ\left(i d \otimes \varphi_{\mathcal{E}}\right): R_{\mathcal{E}}^{*} \otimes_{\mathbb{L}^{*}} \operatorname{MGL}_{k}^{* *}(X) \rightarrow \mathcal{E}^{* *}(X)
\end{gathered}
$$

are isomorphisms for all $X \in \mathbf{S m} / k$, the map $\psi$ induces an isomorphism of cohomology theories on $\mathcal{S H}(k)_{\text {fin }}$. As above, this shows that $\psi$ is an isomorphism of oriented weak commutative ring spectra in $\mathcal{S H}(k)$.

\subsection{Geometrically Landweber exact theories}

We specialize to the setting $S=\operatorname{Spec} k, k$ a field of characteristic 0 . As above, let $(\mathcal{E}, c)$ be a oriented weak commutative ring $T$-spectrum in $\mathcal{S H}(k)$.

As we have noted in Remark 1.5, we have the element $t_{\mathcal{E}} \in \mathcal{E}^{1,1}\left(\mathbb{G}_{m}\right)$ corresponding to the unit $1 \in \mathcal{E}^{0,0}(k)$ under the suspension isomorphism, inducing the group homomorphism

$$
t_{\mathcal{E}}^{1}(X): \mathcal{O}_{X}^{\times}(X) \rightarrow \mathcal{E}^{1,1}(X)
$$

In addition, the $\mathcal{E}^{*, *}(k)$-module structure on $\mathcal{E}^{*, *}(X)$ extends $t_{\mathcal{E}}^{1}(X)$ to a map of $\mathcal{E}^{*, *}(k)$-modules

$$
t_{\mathcal{E}}(X): \mathcal{E}^{2 *, *}(k) \otimes_{\mathbb{Z}} \mathcal{O}_{X}^{\times}(X) \rightarrow \mathcal{E}^{2 *+1, *+1}(X) .
$$

Furthermore, the classifying map $\varphi_{\mathcal{E}, c}:$ MGL $\rightarrow \mathcal{E}$ combined with the product in $\mathcal{E}$ cohomology gives rise to the homomorphism

$$
\varphi_{\mathcal{E}, X}: R_{\mathcal{E}}^{*} \otimes_{R_{\mathrm{MGL}}^{*}} \operatorname{MGL}^{2 *-\epsilon, *}(X) \rightarrow \mathcal{E}^{2 *-\epsilon, *}(X)
$$

for $X \in \mathbf{S m} / k$, natural with respect to morphisms in $\mathbf{S m} / k$. If $\eta$ is a generic point of $X$, we may pass to the limit over Zariski open neighborhoods of $\eta$, giving the homomorphism

$$
\varphi_{\mathcal{E}, \eta}: R_{\mathcal{E}}^{*} \otimes_{R_{\mathrm{MGL}}^{*}} \operatorname{MGL}^{2 *-\epsilon, *}(\eta) \rightarrow \mathcal{E}^{2 *-\epsilon, *}(\eta) .
$$

Remark 3.6. By the Hopkins-Morel-Hoyois theorem [6], the map $\rho_{\mathrm{MGL}}: \mathbb{L}^{*} \rightarrow$ $\mathrm{MGL}^{2 * * *}(k)$ classifying the formal group law $F_{\mathrm{MGL}}$ is an isomorphism; in particular, $\mathrm{MGL}^{2 n, n}(k)=0$ for $n>0$. We henceforth identify $\mathbb{L}^{*}$ and $\mathrm{MGL}^{2 * * *}(k)=R_{\mathrm{MGL}}^{*}$ via $\rho_{\mathrm{MGL}}$ and use $\mathbb{L}^{*}$ and $R_{\mathrm{MGL}}^{*}$ interchangeably.

Definition 3.7. We say that an oriented weak commutative ring $T$-spectrum $(\mathcal{E}, c)$ in $\mathcal{S H}(k)$ is geometrically Landweber exact if for all generic points $\eta \in X \in \mathbf{S m} / k$, the map

$$
\varphi_{\mathcal{E}, \eta}: R_{\mathcal{E}}^{*} \otimes_{R_{\mathrm{MGL}}^{*}} \operatorname{MGL}^{2 *-\epsilon, *}(\eta) \rightarrow \mathcal{E}^{2 *-\epsilon, *}(\eta)
$$

is an isomorphism for $\epsilon=0$ and a surjection for $\epsilon=1$.

As we are assuming the $k$ has characteristic 0 , and hence is perfect, this definition would be equivalent to one requiring the above maps to be isomorphisms for all points $\eta \in Y \in \mathbf{S c h} / k$, as the closure of $\eta$ in $Y$ will contain a dense open subscheme $X$, smooth and quasi-projective over $k$. 
Proposition 3.8. 1. For $\eta \in X \in \mathbf{S m} / k$, let $p_{\eta}: \eta \rightarrow$ Spec $k$ be the structure morphism. Then $p_{\eta}^{*}: \mathrm{MGL}^{2 *, *}(k) \rightarrow \mathrm{MGL}^{2 *, *}(\eta)$ is an isomorphism.

2. For $\eta \in X \in \mathbf{S m} / k$, the map $t_{\mathrm{MGL}}(\eta): \mathrm{MGL}^{2 * * *}(k) \otimes_{\mathbb{Z}} k(\eta)^{\times} \rightarrow \mathrm{MGL}^{2 *+1, *+1}(\eta)$ is an isomorphism.

3. For $Y \in \mathbf{S m} / k, n \in \mathbb{Z}, \mathrm{MGL}^{2 n+a, n}(Y)=0$ for all $a>0$.

Proof. We use the (strongly convergent) Hopkins-Morel spectral sequence [6]

$$
E_{2}^{p, q}(n):=\mathbb{L}^{q} \otimes H^{p-q}(Y, \mathbb{Z}(n-q)) \Longrightarrow \mathrm{MGL}^{p+q, n}(Y) .
$$

Recall that for $Y \in \mathbf{S m} / k, H^{a}(Y, \mathbb{Z}(b))=0$ for $a>2 b$. Thus, $E_{2}^{p, q}(n)=0$ for $p+q>$ $2 n$, and hence $\mathrm{MGL}^{m, n}(Y)=0$ for $m>2 n, Y \in \mathbf{S m} / k$, proving (3).

For $\eta \in X \in \mathbf{S m} / k$, we have $H^{a}(\eta, \mathbb{Z}(b))=0$ for $a>b \geqslant 0$ or $b=0$ and $a \neq 0$ or $b<0$. Thus,

$$
E_{2}^{2 n-q, q}(n)=\mathbb{L}^{q} \otimes H^{2(n-q)}(k, \mathbb{Z}(n-q))=0
$$

if $q \neq n$. Similarly,

$$
E_{2}^{2 n-q-1, q}(n)=\mathbb{L}^{q} \otimes H^{2(n-q)-1}(k, \mathbb{Z}(n-q))=0
$$

if $q \neq n-1$. Since $H^{0}(\eta, \mathbb{Z}(0))=\mathbb{Z}$, and $H^{1}(\eta, \mathbb{Z}(1))=k(\eta)^{\times}$, the surviving non-zero terms contributing to $\mathrm{MGL}^{2 n, n}(\eta)$ and $\mathrm{MGL}^{2 n-1, n}(\eta)$ are

$$
E_{2}^{n, n}(n)=\mathbb{L}^{n} ; n \leqslant 0, \quad E_{2}^{n, n-1}(n)=\mathbb{L}^{n-1} \otimes k(\eta)^{\times} ; n \leqslant 1 .
$$

Similarly, the terms

$$
\begin{gathered}
E_{2}^{n+r, n-r+1}=\mathbb{L}^{n-r+1} \otimes H^{2 r-1}(\eta, \mathbb{Z}(r-1)), E_{2}^{n+r, n-r}=\mathbb{L}^{n-r} \otimes H^{2 r}(\eta, \mathbb{Z}(r)), \\
E_{2}^{n-r, n+r-2}=\mathbb{L}^{n+r-2} \otimes H^{-2 r+2}(\eta, \mathbb{Z}(2-r)), \\
E_{2}^{n-r, n+r-1}=\mathbb{L}^{n+r-1} \otimes H^{-2 r+1}(\eta, \mathbb{Z}(1-r))
\end{gathered}
$$

are all zero for $r \geqslant 2$. The part of the spectral sequence computing $\mathrm{MGL}^{2 n, n}(\eta)$ and $\operatorname{MGL}^{2 n-1, n}(\eta)$ thus degenerates at $E_{2}$, giving isomorphisms $\mathbb{L}^{n} \cong \operatorname{MGL}^{2 n, n}(\eta)$ and $\mathbb{L}^{n-1} \otimes k(\eta)^{\times} \cong \mathrm{MGL}^{2 n-1, n}(\eta)$ for all $n$.

As the spectral sequence is natural in $Y$ and the the pull-back $p_{\eta}^{*}: H^{0}(k, \mathbb{Z}(0)) \rightarrow$ $H^{0}(\eta, \mathbb{Z}(0))$ is an isomorphism, $p_{\eta}^{*}: \mathrm{MGL}^{2 * * *}(k) \rightarrow \mathrm{MGL}^{2 *, *}(\eta)$ is an isomorphism, proving (1).

To complete the proof, we must check that the isomorphisms $\mathbb{L}^{q} \rightarrow \mathrm{MGL}^{2 q, q}(k)$ given above arise from the classifying map $\mathbb{L}^{*} \rightarrow \mathrm{MGL}^{2 *, *}(k)$, and also that the isomorphisms $\mathbb{L}^{q} \otimes k(\eta)^{\times} \rightarrow \mathrm{MGL}^{2 q+1, q+1}(\eta)$ are induced by $t_{\mathrm{MGL}}^{1}(\eta)$ and the isomorphism $\mathbb{L}^{q} \rightarrow \mathrm{MGL}^{2 q, q}(k)$.

The fact that the isomorphisms $\mathbb{L}^{q} \rightarrow \mathrm{MGL}^{2 q, q}(k)$ are the maps coming from the homomorphism $\mathbb{L}^{*} \rightarrow \mathrm{MGL}^{2 *, *}(k)$ classifying the formal group law for $\mathrm{MGL}^{*, *}$ follows directly from construction of the spectral sequence in [6]: the spectral sequence arises from a choice of polynomial generators $x_{1}, x_{2}, \ldots$ for $\mathbb{L}^{*}$, which are then considered as maps $x_{n}: \mathbb{S}_{k} \rightarrow \Sigma^{2 n, n}$ MGL via the classifying map, and these maps are in turn used to construct the tower which gives rise to the spectral sequence (which is then identified with the slice tower for MGL).

Let us now check that the map $t_{\mathrm{MGL}}^{1}(\eta): k(\eta)^{\times} \rightarrow \mathrm{MGL}^{1,1}(\eta)$ is the isomorphism given by the spectral sequence. Since $f_{0} \mathrm{MGL}=\mathrm{MGL}$, we have the distinguished 
triangle $f_{1} \mathrm{MGL} \rightarrow \mathrm{MGL} \stackrel{p_{0}}{\longrightarrow} s_{0} \mathrm{MGL} \rightarrow f_{1} \mathrm{MGL}[1]$. Via the isomorphism $s_{0} \mathrm{MGL} \cong$ $M \mathbb{Z}$, the unit for MGL goes to the unit in $M \mathbb{Z}$, and thus MGL $\rightarrow M \mathbb{Z}$ induces a commutative diagram

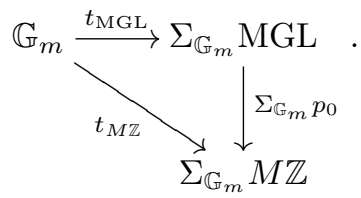

But the spectral sequence $E_{*}^{* *}(1)$ arises by taking the slice tower for MGL and applying $\Sigma_{\mathbb{G}_{m}}$. The spectral sequence computation we have just made shows that the isomorphism $\mathrm{MGL}^{1,1}(\eta) \rightarrow H^{1}(\eta, \mathbb{Z}(1))$ arises from the edge homomorphism associated to the map $\Sigma_{\mathbb{G}_{m}} p_{0}$, which is split by composing the inverse of the isomorphism $t_{M \mathbb{Z}}^{1}: k(\eta)^{\times} \rightarrow H^{1}(\eta, \mathbb{Z}(1))$ with $t_{\mathrm{MGL}}^{1}$. This verifies our assertion for $t_{\mathrm{MGL}}^{1}(\eta)$.

The assertion (2) follows from this and the product structure on the slice spectral sequence (see $[\mathbf{2 2}]$ ).

Our next result shows that passing from a Landweber exact theory $(\mathcal{E}, c)$ to its effective cover $\left(f_{0} \mathcal{E}, c_{0}\right)$ results in a geometrically Landweber exact theory with coefficient ring the evident truncation of the coefficient ring $R_{\mathcal{E}}^{*}$. In fact, it follows directly from the universal property of the slice truncation that $R_{f_{0} \mathcal{E}}^{n}=R_{\mathcal{E}}^{n}$ for $n \leqslant 0$; the vanishing of $R_{f_{0} \mathcal{E}}^{n}$ for $n>0$ is less evident and our proof requires the Hopkins-MorelHoyois spectral sequence.

Theorem 3.9. Let $(\mathcal{E}, c)$ be an oriented weak commutative ring T-spectrum. Suppose that $(\mathcal{E}, c)$ is Landweber exact. Then:

1. Both $(\mathcal{E}, c)$ and the effective cover $\left(f_{0} \mathcal{E}, c_{0}\right)$ are geometrically Landweber exact.

2. The coefficient ring of $f_{0} \mathcal{E}$ is the graded subring of $R_{\mathcal{E}}^{*}$ given by truncation:

$$
R_{f_{0} \mathcal{E}}^{n}= \begin{cases}R_{\mathcal{E}}^{n} & \text { if } n \leqslant 0 \\ 0 & \text { if } n>0\end{cases}
$$

3. The slice spectral sequences for $\mathcal{E}^{*, *}(Y)$ and for $f_{0} \mathcal{E}^{*, *}(Y)$ are strongly convergent for all $Y \in \mathbf{S m} / k$.

Proof. We first show $(3)$. As $f_{q}\left(f_{0} \mathcal{E}\right)=f_{q}(\mathcal{E})$ for $q \geqslant 0$, it suffices to show that slice spectral sequences for $\mathcal{E}^{*, *}(Y)$ is strongly convergent for all $Y \in \mathbf{S m} / k$.

We have already seen that $\operatorname{MGL}^{2 n+a, n}(Y)=0$ for all $Y \in \mathbf{S m} / k, a>0, n \in \mathbb{Z}$. Since $\mathcal{E}$ is Landweber exact, this shows that $\mathcal{E}^{2 n+a, n}(Y)=0$ for all $Y \in \mathbf{S m} / k, a>0$, $n \in \mathbb{Z}$, and thus $\pi_{m+r, r}(\mathcal{E})=0$ for all $m \leqslant r-1, r \in \mathbb{Z}$. We may therefore apply lemma 2.1 with $f$ the function $f(r)=r-1$, giving the convergence.

We now prove (1) and (2) by comparing the slice spectral sequences for $\mathcal{E}$ and $f_{0} \mathcal{E}$. For $\mathcal{F}$ a $T$-spectrum and $Y \in \mathbf{S m} / k$, the $E_{2}$-term is given by

$$
E_{2}^{p, q}(n)(\mathcal{F}, Y)=\left[\Sigma_{T}^{\infty} Y_{+}, \Sigma^{p+q, n} s_{-q} \mathcal{F}\right]_{\mathcal{S H}(k)} .
$$

Since $s_{-q} f_{0} \mathcal{E}=s_{-q} \mathcal{E}$ for $q \leqslant 0$ and $s_{-q} f_{0} \mathcal{E}$ is zero for $q>0$, we have

$$
E_{2}^{p, q}(n)\left(f_{0} \mathcal{E}, Y\right)= \begin{cases}E_{2}^{p, q}(n)(\mathcal{E}, Y) & \text { for } q \leqslant 0 \\ 0 & \text { for } q>0\end{cases}
$$


Since $\mathcal{E} \cong \operatorname{MGL}\left(R_{\mathcal{E}}^{*}\right)$ by Lemma 3.5 , it follows from $[\mathbf{2 4} \text {, Theorem } 6.1]^{3}$ that

$$
E_{2}^{p, q}(n)(\mathcal{E}, Y)=H^{p-q}\left(Y, R_{\mathcal{E}}^{q}(n-q)\right) .
$$

The computation is now essentially the same as for $\mathcal{E}=$ MGL, given in Proposition 3.8: Let $\eta$ be the generic point of some $X \in \mathbf{S m} / k$. Then in the spectral sequence $E_{*}^{* *}(n)$ converging to $\mathcal{E}^{*, n}(\eta)$, we have

$$
E_{2}^{n, n}(n)=R_{\mathcal{E}}^{n}, \quad E_{2}^{n, n-1}(n)=R_{\mathcal{E}}^{n-1} \otimes_{\mathbb{Z}} k(\eta)^{\times}
$$

as the only $E_{2}$ terms contributing to $\mathcal{E}^{2 n, n}(\eta)$ and $\mathcal{E}^{2 n-1, n}(\eta)$, and all differentials entering and leaving these terms are zero. Thus, we have isomorphisms $\mathcal{E}^{2 n, n}(\eta)=R_{\mathcal{E}}^{n}$ and $R^{n-1} \otimes k(\eta)^{\times} \cong \mathcal{E}^{2 n-1, n}(\eta)$. As the $E_{2}$ terms for the spectral sequence converging to $\left(f_{0} \mathcal{E}\right)^{2 n, n}(\eta)$ and $\left(f_{0} \mathcal{E}\right)^{2 n-1, n}(\eta)$ are just a truncation of these $E_{2}$ terms, we have

$$
\left(f_{0} \mathcal{E}\right)^{2 n, n}(\eta)=\left\{\begin{array}{ll}
\mathcal{E}^{2 n, n}(\eta) & \text { for } n \leqslant 0 \\
0 & \text { for } n>0 ;
\end{array}\left(f_{0} \mathcal{E}\right)^{2 n-1, n}(\eta)= \begin{cases}\mathcal{E}^{2 n-1, n}(\eta) & \text { for } n \leqslant 1 \\
0 & \text { for } n>1\end{cases}\right.
$$

Arguing as in the proof of Proposition 3.8, the result follows.

\section{Oriented duality theories}

For the remainder of the paper, we will take $S=\operatorname{Spec} k, k$ a field of characteristic 0 .

Recall from $[\mathbf{1 0}, \S 1]$ the category $\mathbf{S P} / k$ of smooth pairs over $k$, with objects ( $M, X), M \in \mathbf{S m} / k$ and $X \subset M$ a closed subset (not necessarily smooth); a morphism $f:(M, X) \rightarrow(N, Y)$ is a morphism $f: M \rightarrow N$ in $\mathbf{S m} / k$ such that $f^{-1}(Y) \subset X$. For a full subcategory $\mathcal{V}$ of $\mathbf{S c h} / k$, let $\mathcal{V}^{\prime}$ be the subcategory of $\mathcal{V}$ with the same objects as $\mathcal{V}$, but with morphisms the projective morphisms in $\mathbf{S c h} / k$.

Building on work of Mocanasu [13] and Panin [20], we have defined in [10, Definition 3.1] the notion of a bi-graded oriented duality theory $(H, A)$ on $\mathbf{S c h} / k$. Here $A$ is a bi-graded oriented cohomology theory on $\mathbf{S P} / k,(M, X) \mapsto A_{X}^{*, *}(M)$, and $H$ is a functor from $\mathbf{S c h} / k^{\prime}$ to bi-graded abelian groups. The oriented cohomology theory $A$ satisfies the axioms listed in $\left[\mathbf{1 0}\right.$, Definitions 1.2, 1.5]. In particular, $(M, X) \mapsto A_{X}^{* * *}(M)$ admits a long exact sequence

$$
\ldots \rightarrow A_{X}^{*, *}(M) \rightarrow A^{*, *}(M) \rightarrow A^{*, *}(M \backslash X) \stackrel{\partial}{\rightarrow} A_{X}^{*+1, *}(M) \rightarrow \ldots,
$$

where for instance $A^{*, *}(M):=A_{M}^{*, *}(M)$, and the boundary map $\partial$ is part of the data. In addition, there is an excision property and a homotopy invariance property. The ring structure is given by external products and pull-back by the diagonal. The orientation is given by a collection of isomorphisms $\operatorname{Th}_{X}^{E}: A_{X}(M) \rightarrow A_{X}(E)$, for $(M, X) \in \mathbf{S P} / k$ and $E \rightarrow M$ a vector bundle, satisfying the axioms of [20, Definition 3.1.1]. We extend some of the results of $[\mathbf{2 0}]$ in [10, Theorem 1.12, Corollary 1.13] to show that the data of an orientation is equivalent to giving well-behaved pushforward maps $f_{*}: A_{X}(M) \rightarrow A_{Y}(N)$ for $(M, X),(N, Y) \in \mathbf{S P} / k$, with the meaning of "well-behaved" detailed in $[\mathbf{1 0}, \S 1]$.

The homology theory $H$ comes with push-forward maps $f_{*}: H_{*, *}(X) \rightarrow H_{*, *}(Y)$ for $f: X \rightarrow Y$ projective, restriction maps $j^{*}: H_{*, *}(X) \rightarrow H_{*, *}(U)$ for each open immersion $j: U \rightarrow X$ in $\mathbf{S c h} / k$, external products $\times: H_{*, *}(X) \otimes H_{*, *}(Y) \rightarrow H_{*, *}(X \times Y)$,

\footnotetext{
${ }^{3}$ The assumption (SlMGL) in the statement of this result is fulfilled, by the work of Hoyois [6].
} 
boundary maps $\partial_{X, Y}: H_{*, *}(X \backslash Y) \rightarrow H_{*-1, *}(Y)$ for each closed subset $Y \subset X$, isomorphisms $\alpha_{M, X}: H_{*, *}(X) \rightarrow A_{X}^{2 m-*, m-*}(M)$ for each $(M, X) \in \mathbf{S P} / k, m=\operatorname{dim}_{k} M$, and cap product maps

$$
f^{*}(-) \cap: A_{X}^{a, b}(M) \otimes H_{*, *}(Y) \rightarrow H_{*-a, *-b}\left(Y \cap f^{-1}(X)\right)
$$

for $(M, X) \in \mathbf{S P} / k, f: Y \rightarrow X$ a morphism in $\mathbf{S c h} / k$. These satisfy a number of axioms and compatibilities (see $[\mathbf{1 0}, \S 3]$ for details), which essentially say that a structure for $A_{X}^{* * *}(M)$ is compatible with the corresponding structure for $H_{*, *}(X)$ via the isomorphism $\alpha_{M, X}$. Roughly speaking, this is saying that a particular structure for $A_{X}^{*, *}(M)$ depends only on $X$ and not the choice of embedding $X \hookrightarrow M$.

Remark 4.1. Let $L \rightarrow Y$ be a line bundle on some $Y \in \mathbf{S m} / k$ with 0 -section $0: Y \rightarrow$ $L$. For an oriented cohomology theory $A$, one has the element

$$
c_{1}^{A}(L):=0^{*}\left(0_{*}\left(1_{Y}^{A}\right)\right),
$$

where $1_{Y}^{A} \in A^{0}(Y)$ is the unit element. As pointed out in [20, corollary 3.3.8], or as noted in [10, remark 1.17], for line bundles $L, M$ on some $Y \in \mathbf{S m} / k$, the elements $c_{1}(L), c_{1}(M) \in A^{1}(Y)$ are nilpotent and commute with one another, and hence for each power series $F(u, v) \in A^{*}(k)[[u, v]]$ the evaluation $F\left(c_{1}(L), c_{1}(M)\right)$ gives a well-defined element of $A^{*}(Y)$. In addition, the cohomology theory $A$ has a unique associated formal group law $F_{A}(u, v) \in A^{*}(k)[[u, v]]$ with

$$
F_{A}\left(c_{1}^{A}(L), c_{1}^{A}(M)\right)=c_{1}^{A}(L \otimes M)
$$

for all line bundles $L, M$ on $Y \in \mathbf{S m} / k$.

The main example of oriented duality theory $(H, A)$ is given by an oriented weak commutative ring $T$-spectrum $\mathcal{E}$ in $\mathcal{S H}(k)$, assuming $k$ is a field admitting resolution of singularities (e.g., characteristic 0 ), defined by taking

$$
\mathcal{E}_{X}^{a, b}(M):=\operatorname{Hom}_{\mathcal{S H}(k)}\left(\Sigma_{T}^{\infty}(M / M \backslash X), \Sigma^{a, b} \mathcal{E}\right),
$$

that is, the usual bi-graded cohomology with supports. For each $X \in \mathbf{S c h} / k$, choose a closed immersion of $X$ into a smooth $M$ and set $\mathcal{E}_{a, b}^{\prime}(X):=\mathcal{E}_{X}^{2 m-a, m-b}(M)$, where $m=\operatorname{dim}_{k} M$. The fact that $(M, X) \mapsto \mathcal{E}_{X}^{*, *}(M)$ defines an oriented bi-graded ring cohomology theory is proved just as in the case of $\mathcal{E}=$ MGL, which was discussed in $[\mathbf{1 0}, \S 4]$; the main point is Panin's theorem [20, Theorem 3.7.4], which says that an orientation for $\mathcal{E}$ (in the sense of Definition 1.2) defines an orientation in the sense of ring cohomology theories for the bi-graded $\mathcal{E}$-cohomology with supports. The fact that the formula given above for the homology theory $\mathcal{E}_{*, *}^{\prime}$ is well-defined and extends to make $\left(\mathcal{E}_{*, *}^{\prime}(-), \mathcal{E}_{-}^{*, *}(-)\right)$ a bi-graded oriented duality theory is [10, Theorem 3.4].

Remark 4.2. The results of [10] were proven in the setting of an oriented commutative ring $T$-spectrum $\mathcal{E}$, not that of a oriented weak commutative ring $T$-spectrum. However, the constructions and proofs only use values of $\mathcal{E}$-cohomology on finite diagrams of smooth $k$-schemes, and thus only rely on identities modulo phantom maps. The arguments thus remain valid in the larger context of oriented weak commutative ring $T$-spectra. We will henceforth make use of the results of $[\mathbf{1 0}]$ in this wider context without further comment. 
It follows directly from the construction of $\mathcal{E}^{\prime}$ that the assignment $\left(\mathcal{E}, c_{\mathcal{E}}\right) \mapsto\left(\mathcal{E}^{\prime}, \mathcal{E}\right)$ is functorial in the oriented cohomology theory $\left(\mathcal{E}, c_{\mathcal{E}}\right)$; that is, let ch: (MGL, c $\left.\mathrm{MGL}\right) \rightarrow$ $\left(\mathcal{E}, c_{\mathcal{E}}\right)$ be a morphism of oriented weak commutative ring $T$-spectra. Then ch extends canonically to a natural transformation of oriented duality theories

$$
\left(\mathrm{ch}^{\prime}, \mathrm{ch}\right):\left(\mathrm{MGL}^{\prime}, \mathrm{MGL}\right) \rightarrow\left(\mathcal{E}^{\prime}, \mathcal{E}\right) .
$$

\section{Algebraic cobordism and oriented duality theories}

We recall the theory of algebraic cobordism $X \mapsto \Omega_{*}(X), X \in \mathbf{S c h} / k[\mathbf{1 2}]$. For each $X \in \mathbf{S c h} / k, \Omega_{n}(X)$ is an abelian group with generators $(f: Y \rightarrow X), Y \in \mathbf{S m} / k$ irreducible of dimension $n$ over $k$ and $f: Y \rightarrow X$ a projective morphism [12, Lemma 2.5.11]. $\Omega_{*}$ is the universal oriented Borel-Moore homology theory on $\mathbf{S c h} / k[\mathbf{1 2}$, Theorem 7.1.1], where an oriented Borel-Moore homology theory on Sch $/ k$ consists of the data of a functor from $\mathbf{S c h} / k^{\prime}$ to graded abelian groups, external products, first Chern class operators $\tilde{c}_{1}(L): \Omega_{*}(X) \rightarrow \Omega_{*-1}(X)$ for $L \rightarrow X$ a line bundle, and pull-back maps $g^{*}: \Omega_{*}(X) \rightarrow \Omega_{*+d}(Y)$ for each l.c.i. morphism $g: Y \rightarrow X$ of relative dimension $d$. These of course satisfy a number of compatibilities and additional axioms; see $[\mathbf{1 2}, \S 5.1]$ for details.

For an oriented duality theory $(H, A)$ on $\mathbf{S c h} / k$ and $Y$ in $\mathbf{S m} / k$ of dimension $d$ over $k$, the fundamental class $[Y]_{H, A} \in H_{d}(Y)$ is the image of the unit $1_{Y} \in A^{0}(Y)$ under the inverse of the isomorphism $\alpha_{Y}: H_{d}(Y) \rightarrow A^{0}(Y)$. For an oriented Borel-Moore homology theory $B$ on $\mathbf{S c h} / k$, we similarly have the fundamental class $[Y]_{B} \in B_{d}(Y)$ defined by $[Y]_{B}:=p^{*}(1)$, where $1 \in B_{0}(\operatorname{Spec} k)$ is the unit and $p: Y \rightarrow \operatorname{Spec} k$ the structure morphism.

We recall the following result from $[\mathbf{1 0}]$.

Proposition 5.1 ([10, Propositions 4.2, 4.4, 4.5]). Let $k$ be a field admitting resolution of singularities and let $(H, A)$ be a $\mathbb{Z}$-graded oriented duality theory on $\mathbf{S c h} / k$.

1. There is a unique natural transformation $\vartheta_{H}: \Omega_{*} \rightarrow H_{*}$ of functors $\mathbf{S c h} / k^{\prime} \rightarrow$ $\mathbf{G r A b}$, such that $\vartheta_{H}(Y)$ is compatible with fundamental classes for $Y \in \mathbf{S m} / k$. In addition, $\vartheta_{H}$ is compatible with pull-back maps for open immersions in $\mathbf{S c h} / k$, with 1 st Chern class operators, with external products, and with cap products.

2. For $Y \in \mathbf{S m} / k$, the map $\vartheta^{A}(Y): \Omega^{*}(Y) \rightarrow A^{*}(Y)$ induced by $\vartheta_{H}$, the identity $\Omega^{*}(Y)=\Omega_{\operatorname{dim} Y-*}(Y)$ and the isomorphism $\alpha_{Y}: H_{\operatorname{dim} Y-*}(Y) \rightarrow A^{*}(Y)$ is a ring homomorphism and is compatible with pull-back maps for arbitrary morphisms in $\mathrm{Sm} / k$. Finally, one has

$$
\vartheta^{A}(Y)\left(c_{1}^{\Omega}(L)\right)=c_{1}^{A}(L)
$$

for each line bundle $L \rightarrow Y$.

Remark 5.2. We have already noted that one has a formal group law $F_{A}(u, v) \in$ $A^{*}(k)[[u, v]]$ associated to the oriented cohomology theory $A$. Similarly, for each oriented Borel-Moore homology theory $B$ on $\mathbf{S c h} / k$, there is an associated formal group law $F_{B}(u, v) \in B_{*}(k)[[u, v]]$, determined by the identity

$$
F_{B}\left(c_{1}(L), c_{1}(M)\right)=c_{1}(L \otimes M)
$$


for each pair of line bundles $L, M$ on some $Y \in \mathbf{S m} / k$ (this follows from [12, Corollary 4.1.8, Proposition 5.2.1, Proposition 5.2.6]). This gives us the graded ring homomorphism $\varphi_{B}: \mathbb{L}_{*} \rightarrow B_{*}(k)$ classifying the formal group law $F_{B}$.

Finally, we recall that the classifying map $\varphi_{\Omega}: \mathbb{L}_{*} \rightarrow \Omega_{*}(k)$ is an isomorphism $[\mathbf{1 2}$, Theorem 1.2.7].

The following is a direct consequence of proposition 5.1.

Corollary 5.3. Let $\left(\mathcal{E}, c_{\mathcal{E}}\right)$ be pair consisting of a weak commutative ring $T$-spectrum $\mathcal{E} \in \mathcal{S H}(k)$ with orientation $c$, and let $\left(\mathcal{E}_{*, *}^{\prime}, \mathcal{E}^{*, *}\right)$ be the corresponding bi-graded oriented duality theory. There is a unique natural transformation

$$
\vartheta_{(\mathcal{E}, c)}: \Omega_{*} \rightarrow \mathcal{E}_{2 *, *}^{\prime}
$$

of functors $\mathbf{S c h} / k^{\prime} \rightarrow \mathbf{G r} \mathbf{A b}$, such that $\vartheta_{(\mathcal{E}, c)}(Y)$ is compatible with fundamental classes for $Y \in \mathbf{S m} / k$. In addition, $\vartheta_{\left(\mathcal{E}, \mathcal{C}_{\mathcal{E}}\right)}$ is compatible with pull-back maps for open immersions in $\mathbf{S c h} / k$, 1st Chern class operators, external products and cap products. For $Y \in \mathbf{S m} / k$, the map $\vartheta^{\mathcal{E}}(Y): \Omega^{*}(Y) \rightarrow \mathcal{E}^{2 *, *}(Y)$ induced by $\vartheta_{\left(\mathcal{E}, c_{\mathcal{E}}\right)}$ is a ring homomorphism and is compatible with pull-back maps for arbitrary morphisms in $\mathbf{S m} / k$, and satisfies

$$
\vartheta_{(\mathcal{E}, c)}(Y)\left(c_{1}^{\Omega}(L)\right)=c_{1}^{\mathcal{E}}(L)
$$

for each line bundle $L \rightarrow Y$.

Remark 5.4. By [12, Lemma 2.5.11], $\Omega_{*}(X)$ is generated as an abelian group by the cobordism cycles $(f: Y \rightarrow X), Y \in \mathbf{S m} / k$ irreducible, $f: Y \rightarrow X$ a projective morphism. Furthermore, the identity $(f: Y \rightarrow X)=f_{*}\left([Y]_{\Omega}\right)$ holds in $\Omega_{\operatorname{dim} Y}(X)$. Thus, $\vartheta_{\left(\mathcal{E}, c_{\mathcal{E}}\right)}$ is characterized by the formula

$$
\vartheta_{\left(\mathcal{E}, c_{\mathcal{E}}\right)}(f: Y \rightarrow X):=f_{*}^{\mathcal{E}^{\prime}}\left([Y]_{\mathcal{E}^{\prime}, \mathcal{E}}\right) .
$$

We may apply Corollary 5.3 in the universal case: $\mathcal{E}=$ MGL with its canonical orientation. This gives us the natural transformation

$$
\vartheta_{\mathrm{MGL}}: \Omega_{*} \rightarrow \mathrm{MGL}_{2 *, *}^{\prime} \text {. }
$$

Theorem 5.5 ([11, Theorem 3.1]). If $k$ is a field of characteristic 0, then the natural transformation (5.1) is an isomorphism.

This result relies on the Hopkins-Morel spectral sequence; see $[\mathbf{5}, \mathbf{6}]$.

In the course of the proof of theorem 5.5, we proved another result which we will be using here.

Let $X$ be in $\mathbf{S c h} / k$ and let $d=d_{X}:=\max _{X^{\prime}} \operatorname{dim}_{k} X^{\prime}$, as $X^{\prime}$ runs over the irreducible components of $X$. We define $\operatorname{MGL}_{2 *, *}^{\prime(1)}(X)$ by

$$
\operatorname{MGL}_{2 *, *}^{\prime(1)}(X):=\underset{W}{\lim } \operatorname{MGL}_{2 *, *}^{\prime}(W)
$$

as $W$ runs over all (reduced) closed subschemes of $X$ that contain no dimension $d$ generic point of $X ; \Omega_{*}^{(1)}(X)$ is defined similarly. The natural transformation $\vartheta_{\mathrm{MGL}}$ 
gives rise to the commutative diagram

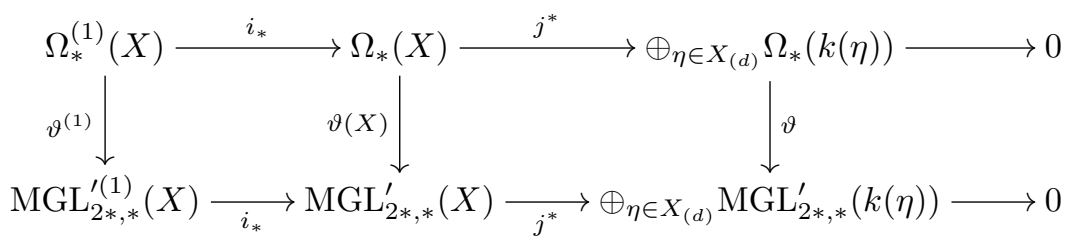

with exact rows and with all vertical arrows isomorphisms. As ( $\left.\mathrm{MGL}^{\prime}, \mathrm{MGL}\right)$ is an oriented duality theory, the bottom line extends to the long exact sequence

$$
\begin{aligned}
\cdots \rightarrow \oplus_{\eta \in X_{(d)}} \mathrm{MGL}_{2 *+1, *}^{\prime}(k(\eta)) & \stackrel{\partial}{\rightarrow} \mathrm{MGL}_{2 *, *}^{\prime(1)}(X) \\
& \stackrel{i_{*}}{\rightarrow} \mathrm{MGL}_{2 *, *}^{\prime}(X) \stackrel{j^{*}}{\rightarrow} \oplus_{\eta \in X_{(d)}} \mathrm{MGL}_{2 *, *}^{\prime}(k(\eta)) \rightarrow 0 .
\end{aligned}
$$

By Proposition 3.8(2), the map

$$
t_{\mathrm{MGL}}(\eta): \mathbb{L}_{*-d+1} \otimes k(\eta)^{\times} \rightarrow \mathrm{MGL}_{2 *+1, *}^{\prime}(k(\eta))
$$

an isomorphism for each $\eta \in X_{(d)}$. We have constructed in $[\mathbf{1 1}, \S 6]$ a group homomorphism

$$
\operatorname{Div}: \mathbb{L}_{*-d+1} \otimes \oplus_{\eta \in X_{(d)}} \mathbb{Z}\left[k(\eta)^{\times}\right] \rightarrow \Omega_{*}^{\prime(1)}(X)
$$

with $\vartheta^{(1)} \circ \operatorname{Div}=\partial \circ \oplus_{\eta} t_{\mathrm{MGL}}(\eta)$. Since the maps $\vartheta^{(1)}$ and $\vartheta(X)$ are isomorphisms, the map Div factors through the surjection

$$
\mathbb{L}_{*-d+1} \otimes \oplus_{\eta \in X_{(d)}} \mathbb{Z}\left[k(\eta)^{\times}\right] \rightarrow \mathbb{L}_{*-d+1} \otimes \oplus_{\eta \in X_{(d)}} k(\eta)^{\times},
$$

and we have the exact sequence

$$
\oplus_{\eta \in X_{(d)}} \mathbb{L}_{*-d+1} \otimes k(\eta)^{\times} \stackrel{\text { Div }}{\longrightarrow} \Omega_{*}^{\prime(1)}(X) \stackrel{i_{*}}{\longrightarrow} \Omega_{*}(X) \stackrel{j^{*}}{\rightarrow} \oplus_{\eta \in X} \Omega_{(d)}(k(\eta)) \rightarrow 0
$$

and the extension of diagram (5.2) to the commutative diagram

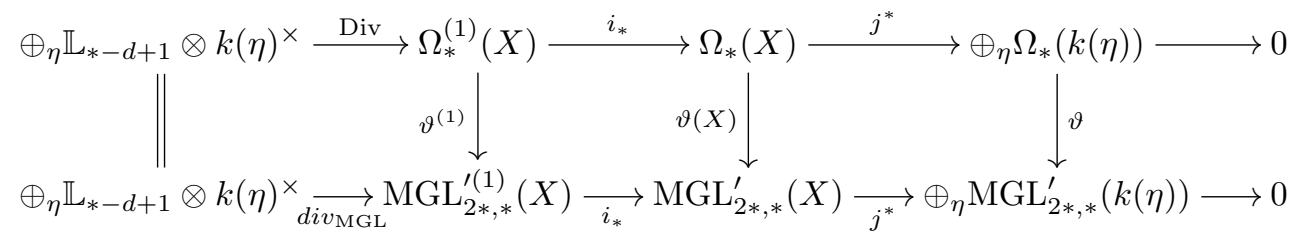

with exact rows and vertical arrows isomorphisms. Here, $\operatorname{div}_{\mathrm{MGL}}:=\partial \circ \oplus_{\eta} t_{\mathrm{MGL}}(\eta)$.

\section{The comparison map}

Let $(\mathcal{E}, c)$ be a weak commutative ring $T$-spectrum in $\mathcal{S H}(k)$ with orientation $c$, and let $\left(\mathcal{E}_{*, *}^{\prime}, \mathcal{E}^{*, *}\right)$ be the corresponding bi-graded oriented duality theory. We have the natural transformation

$$
\vartheta_{(\mathcal{E}, c)}: \Omega_{*} \rightarrow \mathcal{E}_{2 *, *}^{\prime}
$$

given by Corollary 5.3. The map $\vartheta_{(\mathcal{E}, c)}(k)$ makes $R_{*}^{\mathcal{E}}$ an $\Omega_{*}(k)$-algebra; we let $\Omega_{*}^{\mathcal{E}}$ be the oriented Borel-Moore homology theory $\Omega_{*}^{\mathcal{E}}(X):=R_{*}^{\mathcal{E}} \otimes_{\Omega_{*}(k)} \Omega_{*}(X)$. 
As the external products make $\mathcal{E}_{2 *, *}^{\prime}(X)$ an $R_{*}^{\mathcal{E}}$-module and the maps $f_{*}, \tilde{c}_{1}(L)$ are $R_{*}^{\mathcal{E}}$-module maps, we see that $\vartheta_{(\mathcal{E}, c)}$ descends to a natural transformation

$$
\bar{\vartheta}_{(\mathcal{E}, c)}: \Omega_{*}^{\mathcal{E}} \rightarrow \mathcal{E}_{2 *, *}^{\prime}
$$

Lemma 6.1. Suppose that the oriented weak commutative ring T-spectrum $(\mathcal{E}, c)$ is geometrically Landweber exact. Then:

1. For $X \in \mathbf{S c h} / k$ and $\eta \in X$ a point, the $\operatorname{map} \bar{\vartheta}_{\mathcal{E}}: \Omega_{*}^{\mathcal{E}}(\eta) \rightarrow \mathcal{E}_{2 *, *}^{\prime}(\eta)$ is an isomorphism.

2. Take $X$ in $\mathbf{S c h} / k$ and let $j_{i}: \eta_{i} \rightarrow X, i=1, \ldots, r$ be all the generic points of $X$. Then the restriction map $j^{*}: \mathcal{E}_{2 *, *}^{\prime}(X) \rightarrow \oplus_{i=1}^{r} \mathcal{E}_{2 *, *}^{\prime}\left(\eta_{i}\right)$ is surjective.

3. For each generic point $\eta$ of $X$, the map $t_{\mathcal{E}}(\eta): \mathcal{E}^{2 *, *}(k) \otimes_{\mathbb{Z}} k(\eta)^{\times} \rightarrow \mathcal{E}^{2 *+1, *+1}(\eta)$ is surjective.

Proof. For (1), we may replace $X$ with the closure of $\eta$ in $X$, so we may assume that $\eta$ is the generic point of $X$. We note that $\vartheta_{\mathcal{E}}=\varphi_{\mathcal{E}} \circ \vartheta_{\mathrm{MGL}}$. In addition, $\vartheta_{\mathrm{MGL}}$ is an isomorphism, so $\bar{\vartheta}_{\mathcal{E}}(X)$ is up to this isomorphism the same as the map $\mathrm{MGL}_{2 *, *}^{\prime}(X) \otimes_{\mathbb{L}_{*}}$ $R_{*}^{\mathcal{E}} \rightarrow \mathcal{E}_{2 *, *}^{\prime}(X)$. (1) then follows from the hypothesis on $\mathcal{E}$.

For (2), we have the commutative diagram

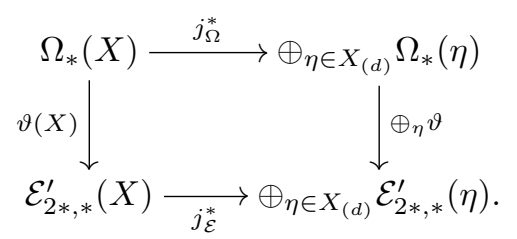

By (1), $\vartheta(\eta): \Omega_{*}^{\mathcal{E}}(\eta) \rightarrow \mathcal{E}_{2 *, *}^{\prime}(\eta)$ is surjective. The map $j_{\Omega}^{*}$ is also surjective, using the right exact localization sequence for $\Omega_{*}$. Thus, the map $j_{\mathcal{E}}^{*}$ is also surjective.

For $(3)$, we can rewrite the map $t_{\mathcal{E}}(\eta)$ as the composition

$$
R_{\mathcal{E}}^{*} \otimes_{\mathbb{L}^{*}} \mathrm{MGL}^{2 * * *}(\eta) \otimes \mathrm{MGL}^{1,1}(\eta) \stackrel{\cup}{\rightarrow} R_{\mathcal{E}}^{*} \otimes_{\mathbb{L}^{*}} \mathrm{MGL}^{2 *+1, *+1}(\eta) \stackrel{\varphi \mathcal{E}, \eta}{\longrightarrow} \mathcal{E}^{2 *+1, *+1}(\eta) .
$$

As the map $\mathrm{MGL}^{2 *, *}(\eta) \otimes \mathrm{MGL}^{1,1}(\eta) \rightarrow \mathrm{MGL}^{2 *+1, *+1}(\eta)$ is surjective, and the map $\varphi_{\mathcal{E}, \eta}$ is surjective by hypothesis, it follows that $t_{\mathcal{E}}(\eta)$ is also surjective.

Theorem 6.2. Suppose that a oriented weak commutative ring $T$-spectrum $(\mathcal{E}, c)$ is geometrically Landweber exact. Then the natural transformation $\bar{\vartheta}_{\left(\mathcal{E}_{, c)}\right)}: \Omega_{*}^{\mathcal{E}} \rightarrow \mathcal{E}_{2 *, *}^{\prime}$ is an isomorphism.

Proof. We write $\bar{\vartheta}$ for $\bar{\vartheta}_{(\mathcal{E}, c)}$. For $\eta$ a point of $X$, the map $\bar{\vartheta}(\eta): \Omega_{*}^{\mathcal{E}}(\eta) \rightarrow \mathcal{E}_{2 *, *}^{\prime}(\eta)$ is an isomorphism by Lemma 6.1(1). In particular, if $X$ has dimension zero over $k$, then $\bar{\vartheta}(X)$ is an isomorphism.

We proceed by induction on the maximum $d$ of the dimensions of the components of $X$; we may assume that $X$ is reduced. We use the constructions and notations from Theorem 5.5 and the discussion following that theorem. We let $\mathcal{E}_{2 *, *}^{\prime(1)}(X)$ be the inductive limit

$$
\mathcal{E}_{2 *, *}^{(1)}(X):=\underset{W}{\underset{W}{\lim }} \mathcal{E}_{2 *, *}^{\prime}(W)
$$

as $W$ runs over all (reduced) closed subschemes of $X$ that contain no dimension $d$ generic point of $X$. This, together with the map Div defined following theorem 5.5 
and the exact localization sequence for $\mathcal{E}_{*, *}^{\prime}$, gives us the commutative diagram with exact rows

$$
\begin{aligned}
& \oplus_{\eta \in X_{(d)}} \mathbb{L}_{*-d+1} \otimes k(\eta) \stackrel{\text { Diy }}{\longrightarrow} \Omega_{*}^{(1)}(X) \stackrel{i_{*}}{\longrightarrow} \Omega_{*}(X) \stackrel{j^{*}}{\longrightarrow} \oplus_{\eta \in X_{(d)}} \Omega_{*}(\eta) \longrightarrow 0 \\
& \vartheta^{(1)} \downarrow \quad \vartheta(X) \downarrow \quad \vartheta \downarrow \\
& \oplus_{\eta \in X_{(d)}} \mathcal{E}_{2 *+1, *}^{\prime}(k(\eta)) \underset{\partial}{\longrightarrow} \mathcal{E}_{2 *, *}^{(1)}(X) \underset{i_{*}}{\rightarrow} \mathcal{E}_{2 *, *}^{\prime}(X) \underset{j^{*}}{\rightarrow} \oplus_{\eta \in X} \mathcal{E}_{(d)} \mathcal{E}_{2 *, *}^{\prime}(\eta) \rightarrow 0 ;
\end{aligned}
$$

the surjectivity in the bottom row comes from lemma 6.1(2).

We apply $R_{*}^{\mathcal{E}} \otimes_{\Omega_{*}(k)}(-)$ to the top row in (6.1). As noted at the beginning of this section, the vertical maps in (6.1) descend to give the commutative diagram

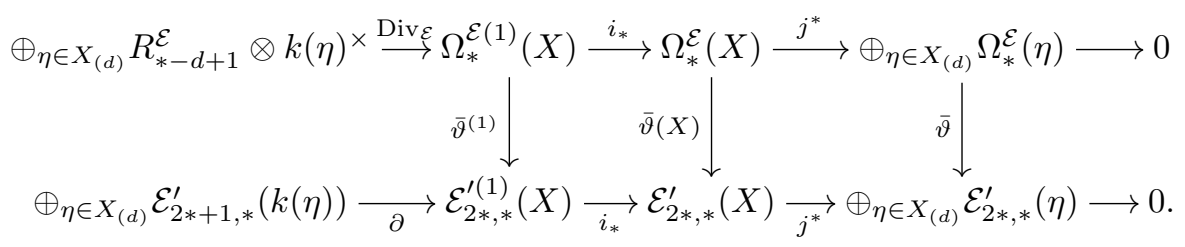

By induction on $d$, the map $\bar{\vartheta}^{(1)}$ is an isomorphism; we have already seen that $\bar{\vartheta}$ is an isomorphism. We note that the bottom row is a sequence of $R_{*}^{\mathcal{E}}$-modules via the the $\mathcal{E}_{2 *, *}^{\prime}(k)$-module structure given by external products.

The universal property of MGL gives the canonical morphism of oriented weak commutative ring $T$-spectra

$$
\varphi_{\mathcal{E}}:\left(\mathrm{MGL}, c_{\mathrm{MGL}}\right) \rightarrow(\mathcal{E}, c),
$$

which extends to the map of bi-graded oriented duality theories

$$
\varphi_{\mathcal{E}}:\left(\mathrm{MGL}_{*, *}^{\prime}, \mathrm{MGL}^{*, *}\right) \rightarrow\left(\mathcal{E}_{*, *}^{\prime}, \mathcal{E}^{*, *}\right) .
$$

As discussed in Remark 1.5, we have for each orientable $\mathcal{E}$ and each $Y \in \mathbf{S m} / k$ the $R_{\mathcal{E}}^{*}$-module map

$$
t_{\mathcal{E}}(Y): \mathcal{O}_{Y}^{\times}(Y) \otimes R_{\mathcal{E}}^{*} \rightarrow \mathcal{E}^{2 *+1, *+1}(Y),
$$

natural in $\mathcal{E}$ and $Y$. This gives us the commutative diagram

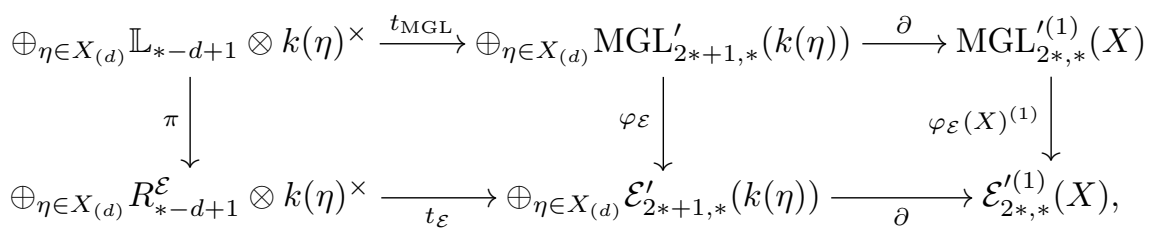

where $\pi$ is induced by the classifying map $\mathbb{L}_{*} \rightarrow R_{*}^{\mathcal{E}}$.

Take $\eta \in X_{(d)}$. By Lemma 6.1(3), the map

$$
t_{\mathcal{E}}(\eta): R_{*-d+1}^{\mathcal{E}} \otimes k(\eta)^{\times} \rightarrow \mathcal{E}_{2 *+1, *}^{\prime}(\eta)
$$

is surjective for each $\eta$. Defining $d i v_{\mathcal{E}}=\partial \circ \sum_{\eta} t_{\mathcal{E}}(\eta)$ and putting this into the diagram 
(6.2) gives us the commutative diagram

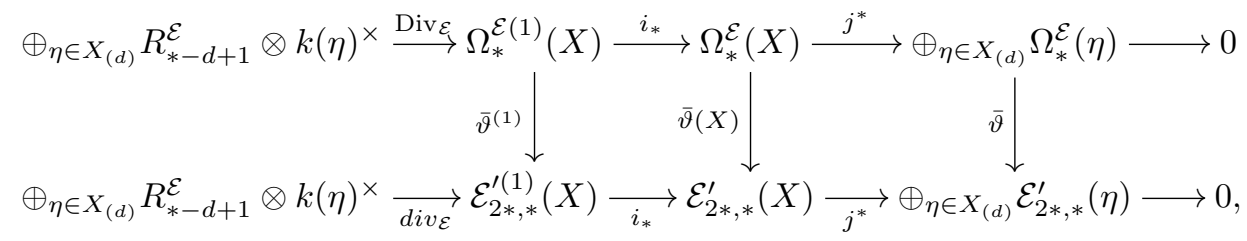

with the bottom row exact and the top row a complex. Recalling that $d i v_{\mathrm{MGL}}=\partial \circ$ $\sum_{\eta} t_{\mathrm{MGL}}(\eta)$, the commutativity of diagram (6.3) gives us the commutative diagram

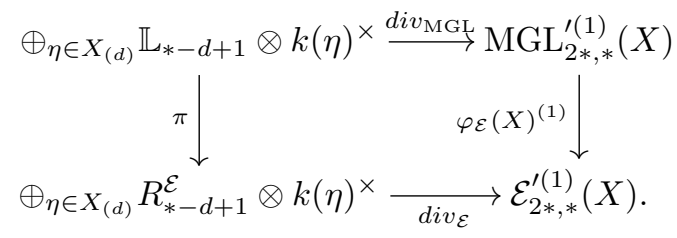

We claim the identity map on $\oplus_{\eta} R_{*-d+1}^{\mathcal{E}} \otimes k(\eta)^{\times}$fills in the diagram (6.4) to a commutative diagram. Assuming this claim, it follows by a diagram chase that the top row is exact and the map $\bar{\vartheta}(X)$ is an isomorphism.

To prove the claim, it follows from the characterization of $\vartheta_{\text {MGL }}$ and $\vartheta_{\mathcal{E}}$ given in Remark 5.4 that $\vartheta_{\mathcal{E}}=\varphi_{\mathcal{E}} \circ \vartheta_{\mathrm{MGL}}$. Thus, patching diagram (6.5) into the left-hand square in the commutative diagram (5.4) yields the commutative diagram

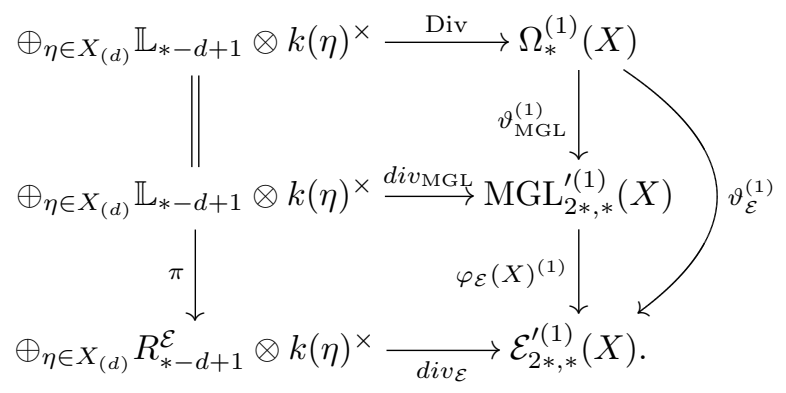

As $\operatorname{Div}_{\mathcal{E}}: R_{*}^{\mathcal{E}} \otimes k(\eta)^{\times} \rightarrow \Omega_{*}^{\mathcal{E}(1)}(X)$ is just the map formed by applying the functor $R_{*}^{\mathcal{E}} \otimes_{\mathbb{L}}(-)$ to Div: $\mathbb{L} \otimes k(\eta)^{\times} \rightarrow \Omega_{*}^{(1)}(X)$, the desired commutativity follows from the commutativity of the outer square in (6.6).

Corollary 6.3. Let $(\mathcal{E}, c)$ be a Landweber exact oriented weak ring T-spectrum in $\mathcal{S H}(k), k$ a field of characteristic 0 , and let $\left(f_{0} \mathcal{E}, c_{0}\right)$ be the effective cover of $(\mathcal{E}, c)$. Then the canonical natural transformations of oriented Borel-Moore homology theories on $\mathbf{S c h}_{k}$

$$
\begin{aligned}
& \vartheta_{\mathcal{E}, c}: R_{\mathcal{E}} \otimes_{\mathbb{L}} \Omega_{*} \rightarrow \mathcal{E}_{2 *, *}^{\prime} \\
& \vartheta_{f_{0}} \mathcal{E}, c_{0}: R_{f_{0} \mathcal{E}} \otimes_{\mathbb{L}} \Omega_{*} \rightarrow f_{0} \mathcal{E}_{2 *, *}^{\prime}
\end{aligned}
$$


are isomorphisms. Moreover, the canonical natural transformations of oriented cohomology theories on $\mathbf{S m} / k$

$$
\begin{aligned}
& \vartheta_{\mathcal{E}, c}: R_{\mathcal{E}} \otimes_{\mathbb{L}} \Omega^{*} \rightarrow \mathcal{E}^{2 *, *} \\
& \vartheta_{f_{0} \mathcal{E}, c_{0}}: R_{f_{0} \mathcal{E}} \otimes_{\mathbb{L}} \Omega^{*} \rightarrow f_{0} \mathcal{E}^{2 *, *}
\end{aligned}
$$

are isomorphisms.

Proof. By Theorem 3.9, both $(\mathcal{E}, c)$ and $\left(f_{0} \mathcal{E}, c_{0}\right)$ are geometrically Landweber exact. We then apply theorem 6.2 to yield the desired isomorphisms of oriented Borel-Moore homology theories.

The statement about the oriented cohomology theories on $\mathbf{S m} / k$ follows by restriction from $\mathbf{S c h} / k$ to $\mathbf{S m} / k$, using the equivalence of oriented Borel-Moore homology theories and oriented cohomology theories on $\mathbf{S m} / k$ [12, Proposition 5.2.1].

\section{References}

[1] K.S. Brown, Abstract homotopy theory and generalized sheaf cohomology, Trans. Amer. Math. Soc. 186 (1973), 419-458.

[2] S. Dai and M. Levine, Connective algebraic K-theory, J. K-Theory 13 (2014), no. $1,9-56$.

[3] B.I. Dundas, M. Levine, P.A. Østvær, O. Röndigs, and V. Voevodsky, Motivic homotopy theory, Lectures from the Summer School held in Nordfjordeid, August 2002, Universitext. Springer-Verlag, Berlin, 2007.

[4] P.G. Goerss, and J.F.Jardine, Localization theories for simplicial presheaves, Canad. J. Math. 50 (1998), no. 5, 1048-1089.

[5] M. Hopkins, and F. Morel, Slices of MGL, lecture (Hopkins), Harvard Univ., Dec. 2, 2004, available as "Week 8" on a webpage of T. Lawson presenting notes from a seminar given by Mike Hopkins, Harvard, fall of 2004, http://www . math. umn.edu/ tlawson/motivic.html

[6] M. Hoyois, From algebraic cobordism to motivic cohomology, J. Reine Angew. Math. 702 (2015), 173-226.

[7] J.F. Jardine, Motivic symmetric spectra, Doc. Math. 5 (2000), 445-553.

[8] M. Levine, A comparison of motivic and classical homotopy theories, J. Topol. 7 (2014), no. 2, 327-362.

[9] M. Levine, The homotopy coniveau tower, J Topology 1 (2008) 217-267.

[10] M. Levine, Oriented cohomology, Borel-Moore homology and algebraic cobordism, Michigan Math. J., Special Issue in honor of Melvin Hochster, Volume 57, August 2008, 523-572.

[11] M. Levine, Comparison of cobordism theories, J. Algebra 322 (2009), no. 9, 3291-3317.

[12] M. Levine and F. Morel, F. Algebraic cobordism, Springer Monographs in Mathematics, Springer, Berlin, 2007.

[13] M. Mocanasu, Borel-Moore functors and algebraic oriented theories, Preprint (2004), K-theory preprint archive 713, http://www.math.uiuc.edu/ K-theory/0713/. 
[14] F. Morel, An introduction to $\mathbb{A}^{1}$-homotopy theory, Contemporary Developments in Algebraic $K$-Theory, 357-441, ICTP Lect. Notes, XV, Abdus Salam Int. Cent. Theoret. Phys., Trieste, 2004.

[15] F. Morel, and V. Voevodsky, $\mathbb{A}^{1}$-homotopy theory of schemes, Inst. Hautes Études Sci. Publ. Math. 90 (1999), 45-143.

[16] A. Neeman, On a theorem of Brown and Adams, Topology 36 (1997), no. 3, 619-645.

[17] A. Neeman, Triangulated categories, Annals of Mathematics Studies, 148, Princeton University Press, Princeton, NJ, 2001.

[18] N. Naumann and M. Spitzweck, Brown representability in $\mathbb{A}^{1}$-homotopy theory, J. K-Theory 7 (2011), no. 3, 527-539.

[19] N. Naumann, M. Spitzweck, and P.A. Østvær, Motivic Landweber exactness, Doc. Math. 14 (2009) 551-593.

[20] I. Panin, Oriented cohomology theories of algebraic varieties, II (After I. Panin and A. Smirnov), Homology, Homotopy Appl. 11 (2009), no. 1, 349-405.

[21] I. Panin, K. Pimenov, and O. Röndigs, A universality theorem for Voevodsky's algebraic cobordism spectrum, Homology, Homotopy Appl. 10 (2008), no. 2, 211-226.

[22] J.P. Pelaez, Multiplicative properties of the slice filtration, Astérisque 335 (2011).

[23] J. Riou, Dualité de Spanier-Whitehead en géométrie algébrique, C. R. Math. Acad. Sci. Paris 340 (2005), no. 6, 431-436.

[24] M. Spitzweck, Slices of motivic Landweber spectra, J. K-Theory 9 (2012), no. 1, 103-117.

[25] G. Vezzosi, Brown-Peterson spectra in stable $\mathbb{A}^{1}$-homotopy theory, Rend. Sem. Mat. Univ. Padova 106 (2001) 47-64.

[26] V. Voevodsky, Open problems in the motivic stable homotopy theory. I, Motives, polylogarithms and Hodge theory, Part I (Irvine, CA, 1998), 3-34, Int. Press Lect. Ser., 3, I, Int. Press, Somerville, MA, 2002.

[27] V. Voevodsky, A possible new approach to the motivic spectral sequence for algebraic K-theory, Recent progress in homotopy theory (Baltimore, MD, 2000) 371-379, Contemp. Math. 293 Amer. Math. Soc., Providence, RI, 2002.

[28] V. Voevodsky, $\mathbb{A}^{1}$-homotopy theory, Proceedings of the International Congress of Mathematicians, Vol. I (Berlin, 1998), Doc. Math. 1998, Extra Vol. I, 579-604.

Marc Levine marc.levine@uni-due.de

Universität Duisburg-Essen, Fakultät Mathematik, Thea-Leymann-Straße 9, 45127 Essen, Germany 